Network Working Group

Request For Comments: 1841

Category: Informational
J. Chapman

Cisco Systems, Inc.

D. Coli

Cisco systems, Inc.

A. Harvey

Cisco Systems, Inc.

B. Jensen

Cisco systems, Inc.

K. Rowett

Cisco systems, Inc.

September 1995

PPP Network Control Protocol for LAN Extension

Status of Memo

This memo provides information for the Internet community. This memo does not specify an Internet standard of any kind. Distribution of this memo is unlimited.

Abstract

Telecommunications infrastructure is improving to offer higher bandwidth connections at lower cost. Access to the network is changing from modems to more intelligent devices. This informational RFC discusses a PPP Network Control Protocol for one such intelligent device. The protocol is the LAN extension interface protocol.

Table of Contents

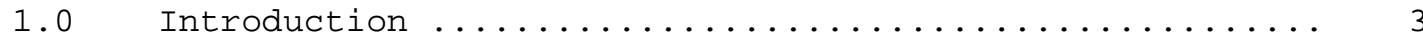

1.1 LAN Extension Interface Topology ................. 4

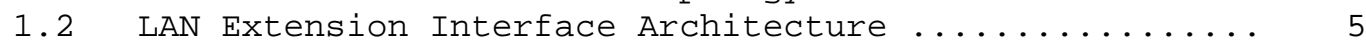

1.3 LAN Extension Interface Protocol ................ 6

2.0 LAN Extension Interface Protocol Control Packets...... 8

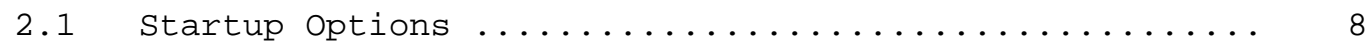

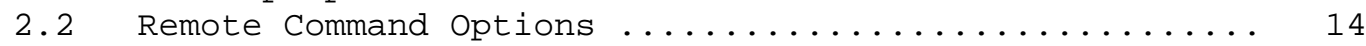

2.3 Conditions for Sending PPP-LEX Packet ............. 17

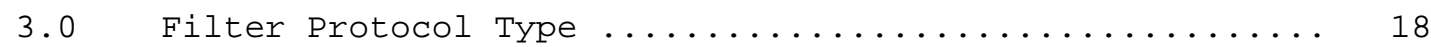

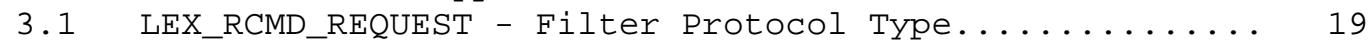

3.2 Response Packets - Filter Protocol Type............ 21

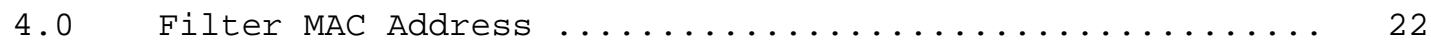

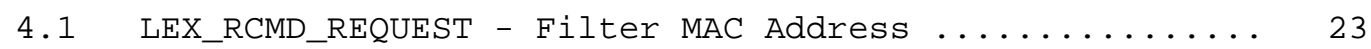

4.2 Response Packets - Filter MAC Address............. 25 


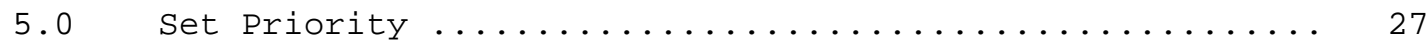

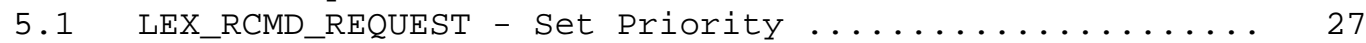

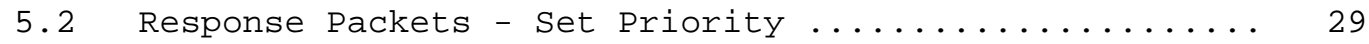

6.0 Disable LAN Extension Ethernet Interface .......... 30

6.1 LEX_RCMD_REQUEST - Disable LAN Extension

Ethernet Interface ............................. 31

6.2 Response Packets - Disable LAN Extension

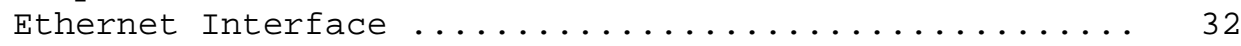

7.0 Enable LAN Extension Ethernet Interface .......... 33

7.1 LEX_RCMD_REQUEST - Enable LAN Extension

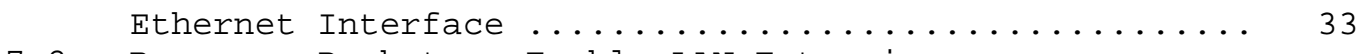

7.2 Response Packets - Enable LAN Extension

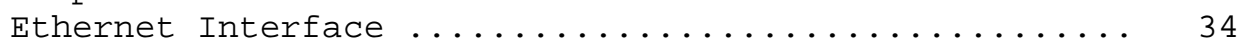

8.0 Reboot LAN Extension Interface Unit .............. 35

8.1 LEX_RCMD_REQUEST - Reboot LAN Extension Interface

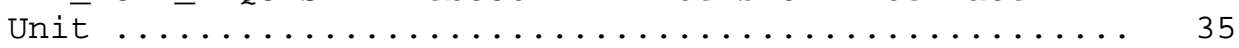

8.2 Response Packets - Reboot LAN Extension

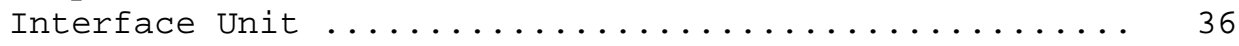

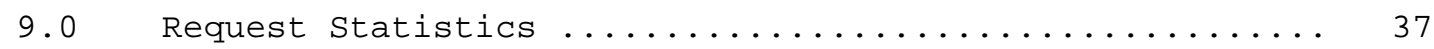

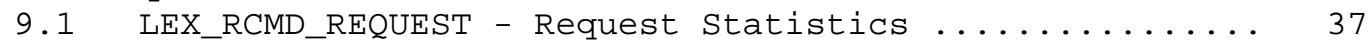

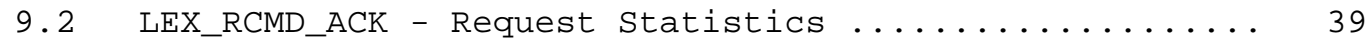

9.3 LEX_RCMD_NAK/LEX_RCMD_REJ - Request Statistics ...... 44

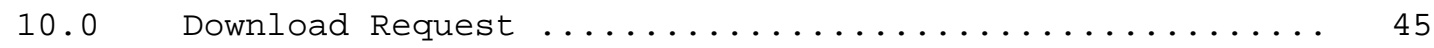

10.1 LEX_RCMD_REQUEST - Download Request ............. 46

10.2 Response Packets - Download Request............. 48

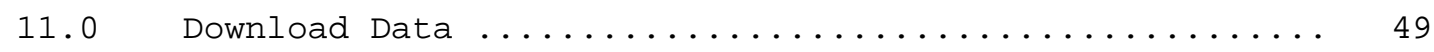

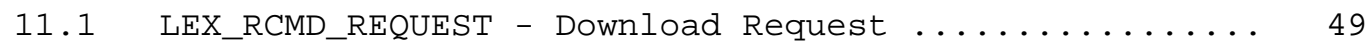

11.2 Response Packets - Download Data ............... 51

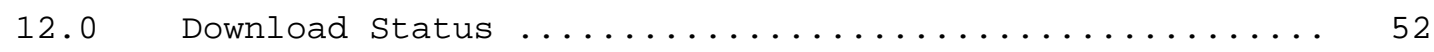

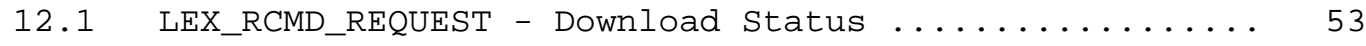

12.2 LEX_RCMD_ACK - Download Status ................ 54

12.3 LEX_RCMD_NAK/LEX_RCMD_REJ - Download Status ...... 56

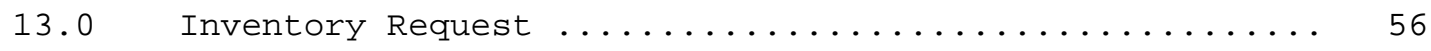

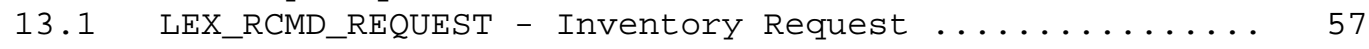

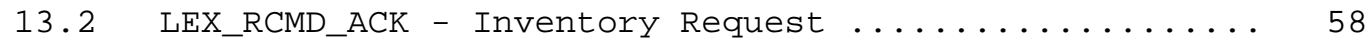

13.3 LEX_RCMD_NAK/LEX_RCMD_REJ - Inventory Request ...... 61

14.0 LAN Extension Interface Protocol Data Packets ....... 62

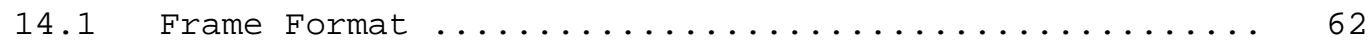

14.2 Summary Field Descriptions................... 63 


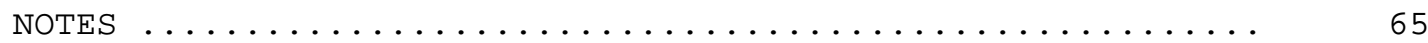

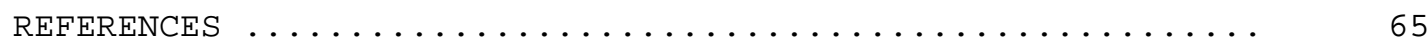

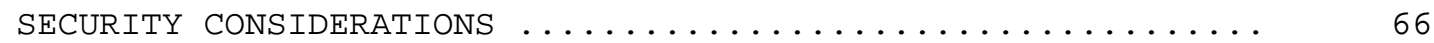

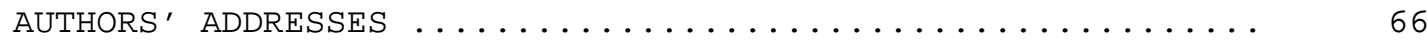

\subsection{Introduction}

An increasing number of corporations allow their employees to telecommute to work due to local government regulations on traffic and air pollution. Additionally, many businesses are run out of internetworked home offices and small branch offices. With these changes in the workplace, more people and businesses require Internet access from small LANs.

Today, routers serve the LAN-to-LAN traffic using high-speed WAN links such as leased lines, ISDN, or Frame Relay. This new breed of Internet users from home offices and small branch offices may have a different, less network-literate skill set than those connecting up to the Internet today. These new users need an alternative to the complex and hard-to-configure routers currently employed for connectivity. One such alternative is a LAN extension interface unit.

A LAN extension interface unit is a hardware device installed at remote sites (such as a home office or small branch office) that connects a LAN across a WAN link to a router at a central site. The following sections introduce a LAN extension interface topology, architecture, and protocol. 


\subsection{LAN Extension Interface Topology}

Figure 1 shows the topology of LAN extension interfaces. The figure shows two LAN extension interface units connected via a WAN link to a central or "host router."

Figure 1 LAN Extension Interface Topology

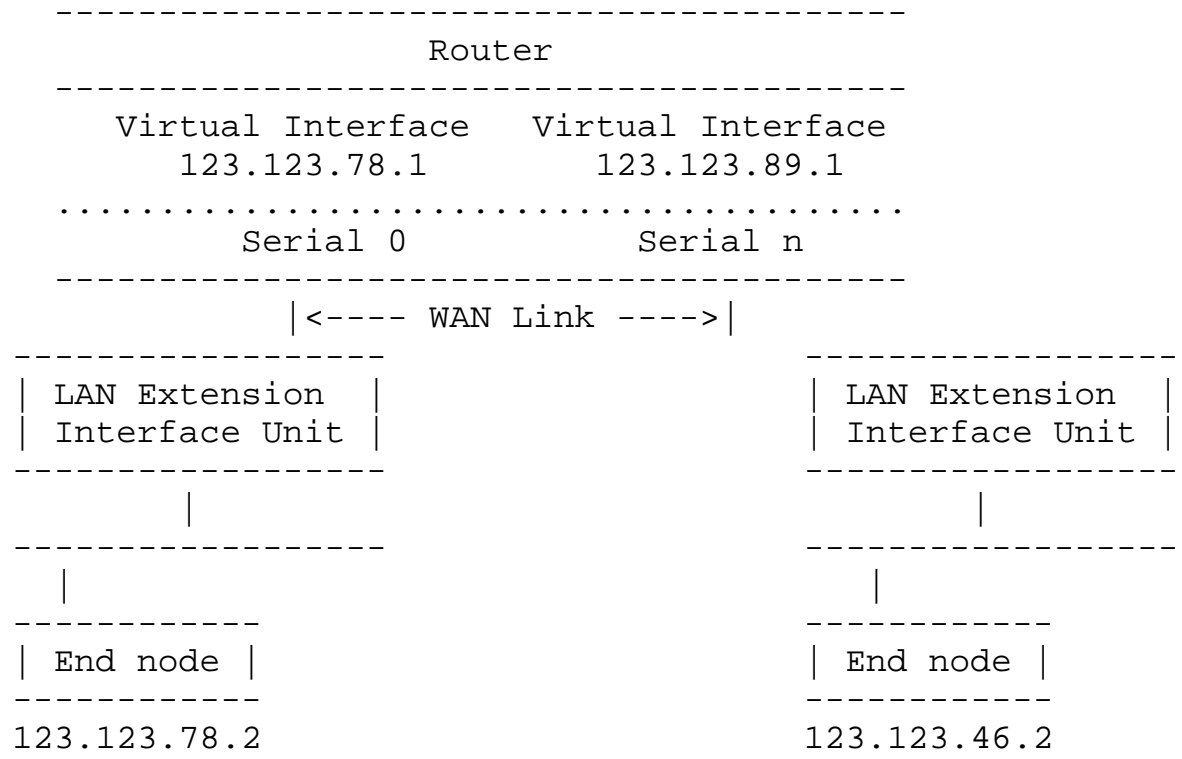

Each LAN extension interface unit maps to a virtual interface at the host router. The virtual interface mirrors the characteristics of the LAN extension interface unit. To the routing protocols, the virtual interface looks just like a local interface, but with the bandwidth of a serial line. The virtual interface keeps the state (up or down) of the LAN extension interface unit, and identifies each LAN extension interface unit by its MAC address.

A LAN extension interface protocol transfers MAC frames from the LAN extension interface unit across the serial line to the host router. At the termination point in the router, the router routes the packets. This topology uses only one subnet per remote LAN rather than two, as is the case when routers exist on both ends of a WAN link. Figure 1 shows this subnetting structure. The IP addresses of the virtual interfaces on the router are in the same subnet as the IP addresses of the end nodes on the LAN of the LAN extension interface unit. The LAN extension interface unit itself has no IP address. 
LAN extension interface units resemble bridges, but with the following distinct differences:

* LAN extension interface units always depend on a host router. They cannot operate standalone or even back-to-back with other LAN extension interface units.

* LAN extension interface units need not employ any spanning tree algorithm.

* (LAN extension interface units transfer MAC frames across a serial line (like bridges), but a router can either route or bridge the LAN extension interface data packets.

\subsection{LAN Extension Interface Architecture}

Figure 2 shows the basic LAN extension interface architecture.

Figure 2 LAN Extension Interface Architecture

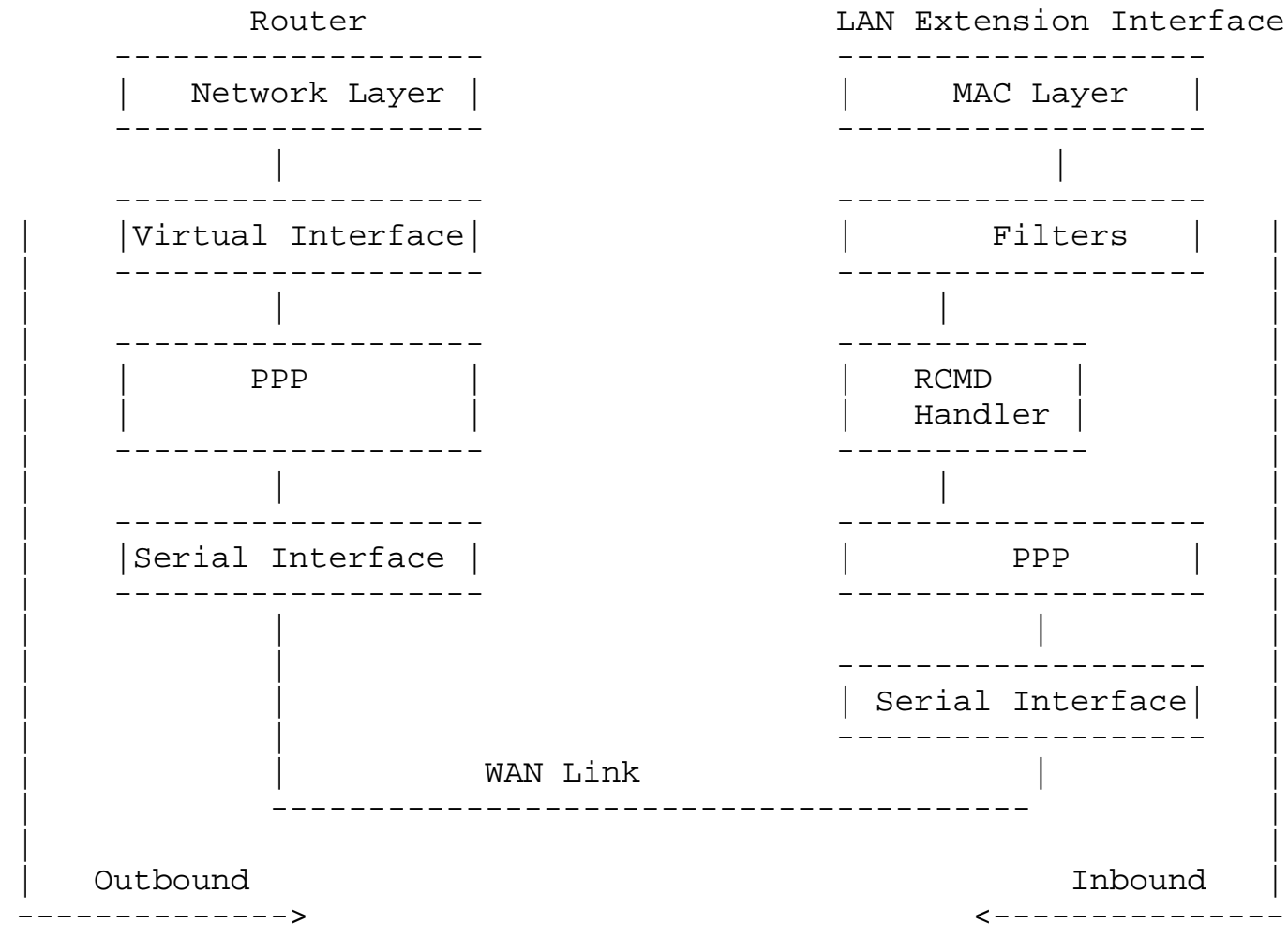


In the inbound direction (from the remote LAN, to the LAN extension interface unit, across the WAN link, to the host router), the LAN extension interface unit can filter received frames to optimize WAN utilization. The LAN extension interface unit can filter frames by protocol type or by MAC address. Frames that pass through the LAN extension interface filters go to the WAN protocol state machine. In Figure 2, this state machine is PPP. The LAN extension interface unit adds PPP encapsulation and forwards the packet to the router via the WAN serial link.

Upon receiving the frame, the host router decapsulates the PPP header and passes the packet to the virtual interface. From there the virtual interface handles the packet like any packet received on a local interface -- by routing or bridging the packet to another interface, depending on configuration.

In the outbound direction (from the host router, across the WAN link, to the LAN extension interface unit, to the LAN), the host router's virtual interface builds the full MAC header, before adding PPP encapsulation. The router then sends the packet across the WAN serial link to the LAN extension interface unit. The LAN extension interface unit strips the PPP header and forwards the packet directly onto the LAN. The host router has already determined that the packet needs to be forwarded to the LAN extension interface unit, hence there is no need for additional filtering or processing at that end.

Embedded in the data stream is a control stream for configuring and managing the LAN extension interface unit from the host router. The virtual interface makes the remote LAN extension interface unit appear like a local router interface to the routing protocols.

Configuration commands and statistics gathering commands are issued on the router to the virtual interface. The virtual interface formats the LAN extension interface remote commands into encoded messages and transfers them in-band with the data packets. The LAN extension interface unit decodes the remote commands and executes them. Responses are similarly formatted messages sent by the LAN extension interface unit to the host router. The remote command messages use a different encapsulation type than the data packets, as described later in this document in the "LAN Extension Interface Protocol Control Packets" and "LAN Extension Interface Protocol Data Packets" sections.

\subsection{LAN Extension Interface Protocol}

To accommodate this LAN extension interface architecture, a new Network Control Protocol (NCP) for PPP exists. This NCP is called PPP-LEX. The basic functionality of PPP-LEX is to encapsulate LAN 
extension interface control and data packets. The IETF has assigned two new protocol types for these functions, as shown in Table 1.

Table 1 IETF Protocol Types for PPP-LEX

Protocol Type $\quad$ Function

0x8041 Encapsulates control packets

0x0041 Encapsulates data packets (MAC frames)

PPP is a natural choice for a LAN extension interface protocol because it allows for negotiating a specific control protocol and options at connection time. This means that network administrators do not have to statically configure the router interface for remote interfaces. Rather, remote interfaces negotiate the link at connection time.

The LAN extension interface protocol employs PPP link operation as described in RFC 1331, which reads as follows:

In order to establish communications of a point-to-point link, each end of the PPP link must first send [Link Control Protocol] LCP packets to configure and test the data link. After the link has been established, the peer may be authenticated. Then PPP must send NCP packets to choose and configure one or more network layer protocols. Once each of the chosen network-layer protocols has been configured, datagrams from each network layer protocol can be sent over the link.

The link will remain configured for communications until explicit LCP or NCP packets close the link down, or until some external event occurs (an inactivity timer expires or network administrator intervention). (References, [1])

Thus, the LAN extension interface unit and the host router exchange PPP-LCP packets at connection time to dynamically configure and test the WAN serial link. Once the link reaches an "opened" state, the LAN extension interface unit and host router exchange PPP-LEX NCP packets to configure the LAN extension interface protocol. Once it is configured, the NCP (PPP-LEX) reaches an "opened" state, and PPP carries the PPP-LEX control and data packets across the serial link. At this point, link traffic is a any combination of LCPs, PPP-LEX NCPs, PPP-LEX control packets, and PPP-LEX data packets.

Note that the LAN extension interface protocol is not a bridging protocol. The only similarity to the PPP Bridging Control Protocol (References. [2]) is that the LAN extension interface protocol also encapsulates MAC frames. 
The following sections detail PPP-LEX control packets and data packets.

\subsection{LAN Extension Interface Protocol Control Packets}

There are two types of PPP-LEX control packets, as follows:

* Startup options packet

* Remote command options packets

The startup options packet is the first PPP-LEX NCP packet that the LAN extension interface unit sends to the host router after the LCP has reached an "opened" state. This required startup options packet configures the LAN extension interface protocol and puts the PPP-LEX NCP in an "opened" state.

Remote command options are the PPP-LEX NCP packets that control the functioning and statistics gathering of the LAN extension interface protocol.

\subsection{Startup Options}

The LAN extension interface unit sends a startup options packet to the host router to negotiate the following startup options:

* MAC Type

* MAC Address

* LAN Extension

The MAC Type startup option informs the host router of the type of media that the LAN extension interface unit is connected to. For example, the LAN extension interface unit may be connected to an Ethernet LAN or a Token Ring LAN. Currently, only Ethernet is supported. The MAC type tells the host router what type of traffic the LAN extension interface unit is prepared to receive. If the host router rejects the MAC type, the LAN extension interface unit sends the Configure-Request again.

The MAC Address startup option sends the MAC address of the LAN extension interface unit to the host router to authenticate the LAN extension interface unit and bind it to the corresponding virtual interface at the host router. The host router also inserts the MAC address in outbound packets. The MAC address is represented in IEEE 802.3 canonical format.

The LAN Extension startup option establishes the network layer protocol (NCP) as PPP-LEX and provides the host router with the LAN extension interface protocol version number. 
Each startup option is transmitted in a series of three fields: Option-Type, Option-Length, and Option-Data fields. The fields are concatenated in the startup options Configure-Request packet.

\section{Frame Format}

Figure 3 shows a summary of the frame format for the startup options packet. The LAN extension interface unit sends this startup options packet to the host router. The LAN extension interface unit transmits these fields from left to right.

Figure 3 Startup Options Frame Format (Configure-Request)

PPP Header

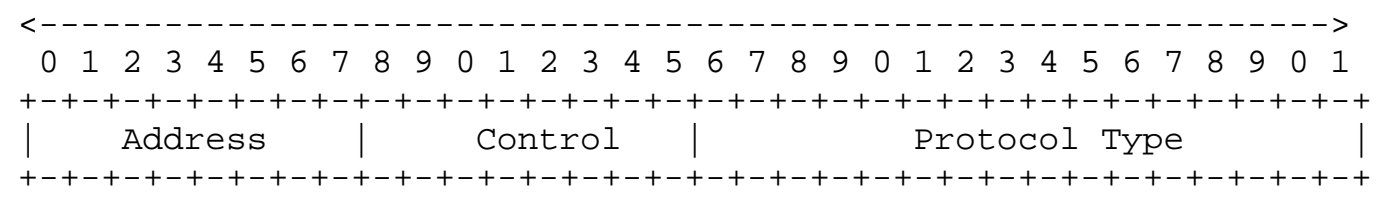

(1 octet)

LAN Extension Interface Protocol Header

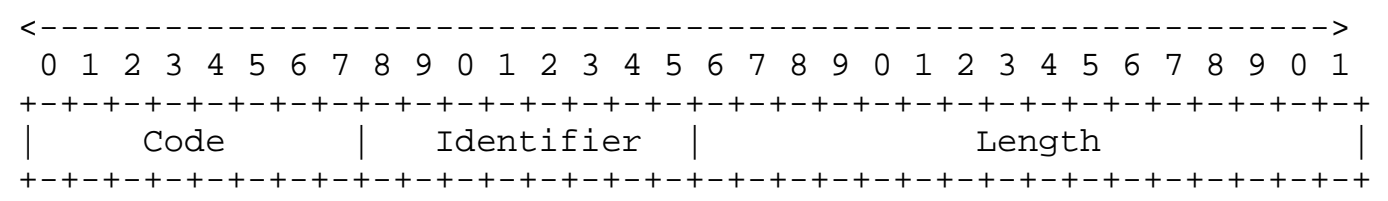

(1 octet)

(1)

(2)

LAN Extension Interface Startup Options

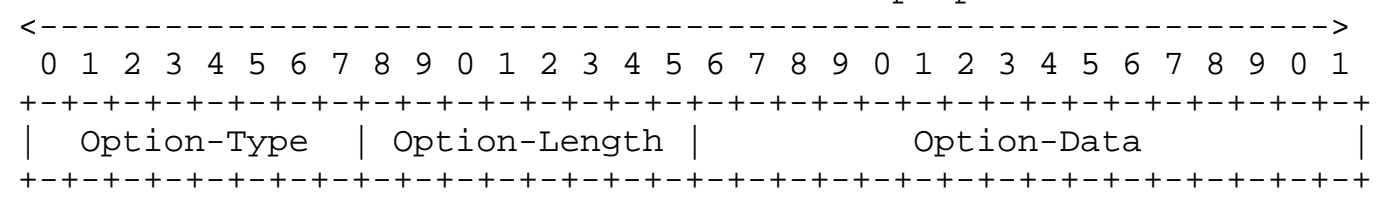

(1 octet)

(1)

Address

This PPP-specified field is one octet and contains the binary sequence 11111111 (hexadecimal 0xFF), the All-Stations address. PPP does not assign individual station addresses. The All-Stations address must be recognized and received by all devices. For more information on this field, refer to "The Point-to-Point Protocol (PPP) for the Transmission of Multi-protocol Datagrams over Pointto-Point Links." (References, [1]) 
Control

This PPP-specified field is one octet and contains the binary sequence 00000011 (hexadecimal 0x03), the Unnumbered Information (UI) command with the $\mathrm{P} / \mathrm{F}$ bit set to zero.

For more information on this field, refer to "The Point-to-Point Protocol (PPP) for the Transmission of Multi-protocol Datagrams over Point-to Point Links." (References, [1])

Protocol-Type

The Protocol-Type field is two octets and contains the IETF-assigned protocol type value. Valid LAN extension interface protocol type values are as follows:

* 0x8041 (for control packets)

* 0x0041 (for data packets)

Because the startup options packet encapsulates LAN extension interface control data, the valid value for this field is 0x8041.

Code

The Code field is one octet and identifies the type of LCP packet that the LAN extension interface packet is sending. Valid values are as follows:

* 0x01 - Configure-Request

* 0x02 - Configure-Ack

* 0x03 - Configure-Nak

* $0 \times 04-$ Configure-Rej

The LAN extension interface unit initiates the startup options packet; therefore, the valid value for this field is 1.

Identifier

The Identifier field is one octet and contains a randomly generated value. The value aids matching requests and replies. It is recommended that a non-zero value be used for the identifier. That is, zero could be used in the future for unsolicited messages from the LAN extension interface unit. Valid values are 0x01-0xFF. 
Length

The Length field is two octets and indicates the length of the entire packet in octets, including the Code, Identifier, Length, and startup options fields.

Option-Type

The Option-Type field is one octet and identifies the startup option being negotiated. Valid values are as follows:

* 0x01 - MAC Type

* 0x03 - MAC Address

* 0x05 - LAN Extension

Option-Length

The Option-Length field is one octet and specifies the length of the startup option fields, including the Option-Type, Option-Data, and Option-Length fields.

Option-Data

The Option-Data field contains the data relating to the value specified in the Option-Type field. That is, if the Option-Type field specifies MAC type (0x01), then the Option-Data field contains the MAC type (Ethernet, Token Ring, and so on). If the Option-Type field specifies MAC address $(0 \times 03)$, then the Option-Data field contains the actual MAC address. If the Option-Type field specifies LAN Extension (0x05), then the Option-Data field contains LAN extension interface software information. The following table defines the contents of the Option-Data field for each possible option-Type field value:

Option-Type Field Value Option-Data

0x01 (MAC Type)

The most up-to-date value of the MAC type as specified in the most recent "Assigned Numbers" RFC. The current valid value from that RFC follows:

* 0x01: IEEE 802.3/Ethernet with canonical addresses

0x03 (MAC Address)

The burned-in MAC address in IEEE 802.3 canonical format.

0x05 (LAN Extension) The LAN extension interface protocol version number. $0 \times 01$ is the current protocol version supported. 


\section{Example}

In the Configure-Request packet that it sends to the host router, the LAN extension interface unit concatenates the option-Type, OptionData, and Option-Length fields for each startup option, as shown in Figure 4. The LAN extension interface unit transmits these fields from left to right.

\section{Figure 4 Sample Startup Options Configure-Request Packet}

PPP Header

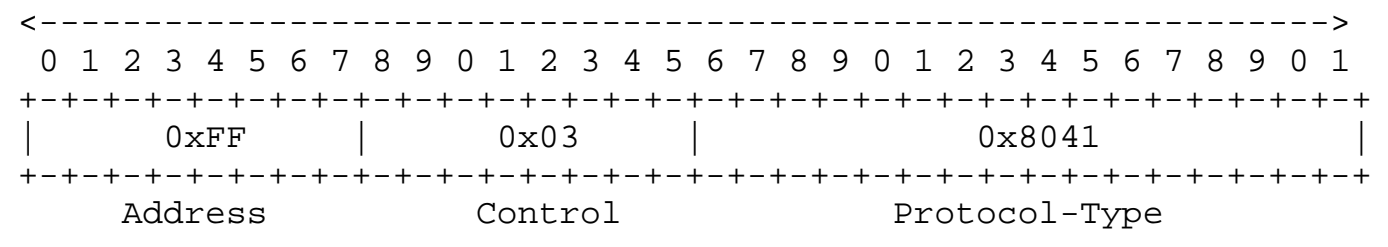

LAN Extension Interface Protocol Header

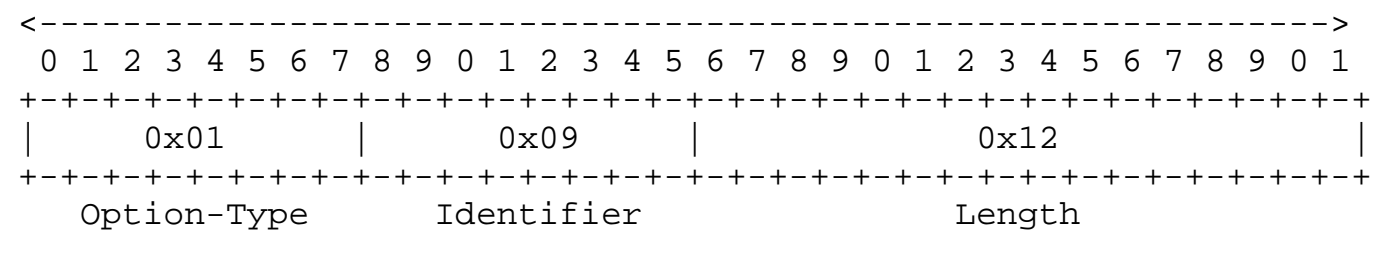

LAN Extension Interface Startup Options

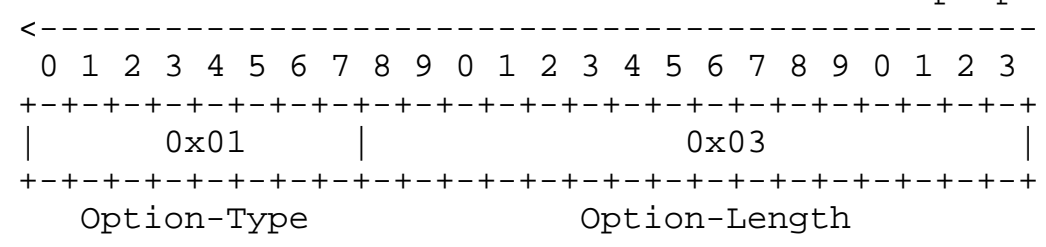

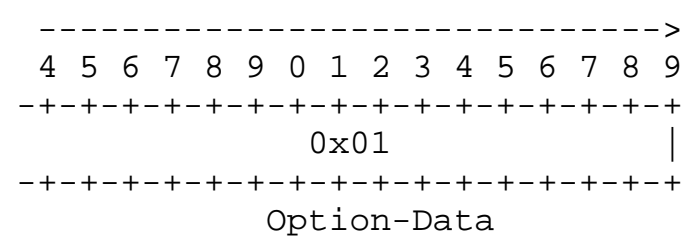


$\begin{array}{llllllllllllllllllllllll}0 & 1 & 2 & 3 & 4 & 5 & 6 & 7 & 8 & 9 & 0 & 1 & 2 & 3 & 4 & 5 & 6 & 7 & 8 & 9 & 0 & 1 & 2 & 3\end{array}$

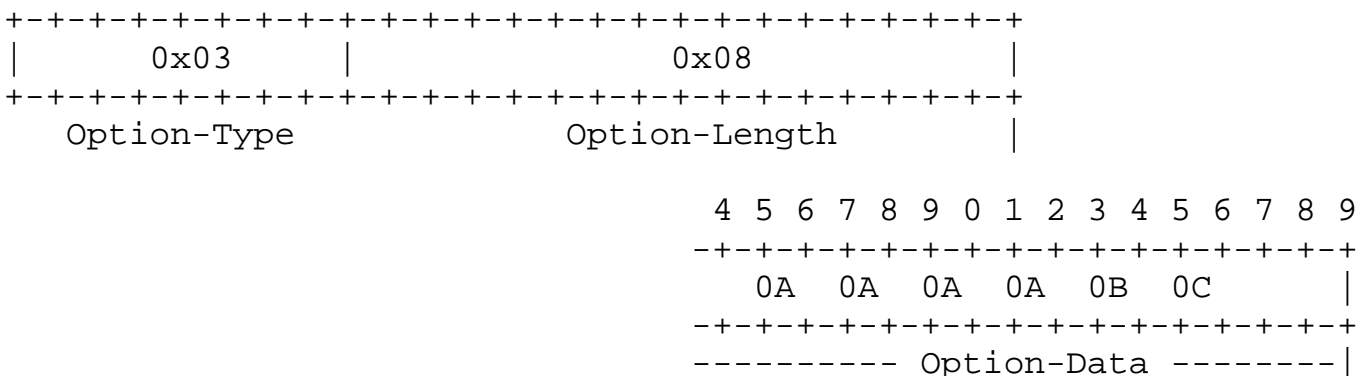

$\begin{array}{llllllllllllllllllllllll}0 & 1 & 2 & 3 & 4 & 5 & 6 & 7 & 8 & 9 & 0 & 1 & 2 & 3 & 4 & 5 & 6 & 7 & 8 & 9 & 0 & 1 & 2 & 3\end{array}$

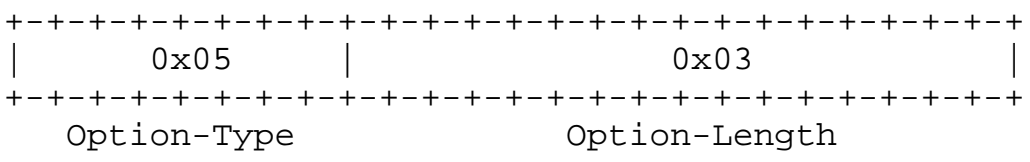

$\begin{array}{llllllllllllllll}4 & 5 & 6 & 7 & 8 & 9 & 0 & 1 & 2 & 3 & 4 & 5 & 6 & 7 & 8 & 9\end{array}$

$-+-+-+-+-+-+-+-+-+-+-+-+-+-+-+-+$ $0 \times 01$

$-+-+-+-+-+-+-+-+-+-+-+-+-+-+-+-+$

Option-Data

In Figure 4, the Address field always contains 0xFF, and the Control field always contains 0x03. The Protocol-Type field value is 0x8041 because the startup options packet is a LAN extension interface control packet. The Code field value is 0x01 because the LAN extension interface unit is sending an LCP Configure-Request packet to configure or negotiate PPP-LEX. The Identifier field contains a randomly generated number. The Length field gives the total length of the entire packet.

The first startup option is the MAC Type startup option. The OptionType value for MAC Type is 0x01. The Option-Length field value for the MAC type startup option is 3 octets. The Option-Data field value is 0x01 because, in this example, the LAN extension interface unit connects to an Ethernet LAN using 802.3 canonical addresses.

The next startup option transmitted is the MAC Address. Its OptionType field value is 0x03, its Option-Length field value is 8 octets, and its Option-Data field value is the actual MAC address.

Lastly, the LAN Extension startup option is transmitted. Its OptionType field value is 0x05, its Option-Length field value is 3 octets, and its Option-Data field value is the LAN extension interface protocol version number $(0 \times 01)$. 
The host router responds to this LCP Configure-Request packet with an LCP Configure-Ack packet, Configure-Nak packet, or Configure-Rej packet. For more information on these packets, refer to "The Pointto-Point Protocol (PPP) for the Transmission of Multi-protocol Datagrams over Point-to-Point Links" RFC. (References, [1])

\subsection{Remote Command Options}

Once the host router responds to the startup options ConfigureRequest packet with a Configure-Ack packet, the PPP-LEX NCP is in an "opened" state, and the LAN extension interface unit and the host router freely exchange PPP-LEX data packets and remote command options packets.

The host router initiates PPP-LEX remote command options packets to control the configuration of the LAN extension interface unit and to gather statistics. There are 11 types of remote command options that the host router can send in a LEX_RCMD_REQUEST packet to the LAN extension interface unit. The LAN extension interface unit responds to a LEX_RCMD_REQUEST packet with a LEX_RCMD_ACK, LEX_RCMD_NAK, or LEX_RCMD_REJ packet.

Frame Format

Figure 5 shows a summary of the frame format for a remote command options packet. These fields are transmitted from left to right.

Figure 5 Remote Command Options Frame Format

PPP Header

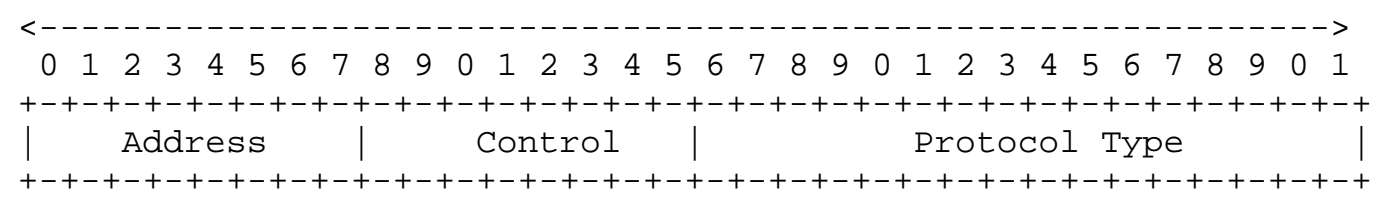

(1 octet)

(2)

LAN Extension Interface Protocol Header

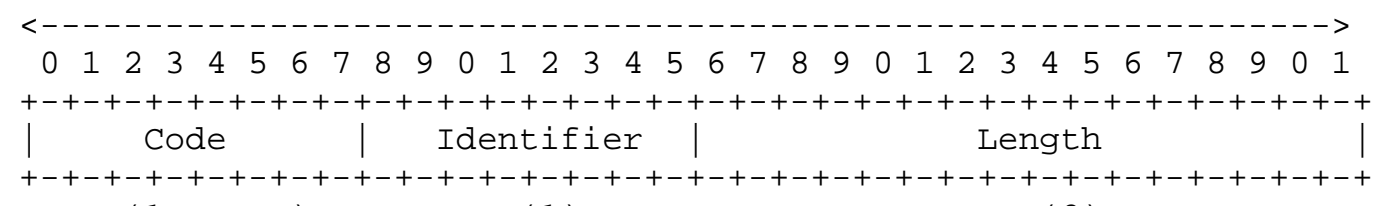

(1 octet)

(1)

(2) 
LAN Extension Interface Remote Command Options

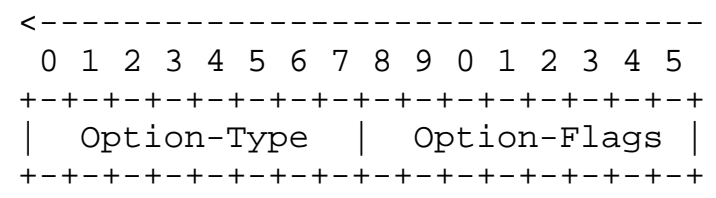

(1 octet)

(1)

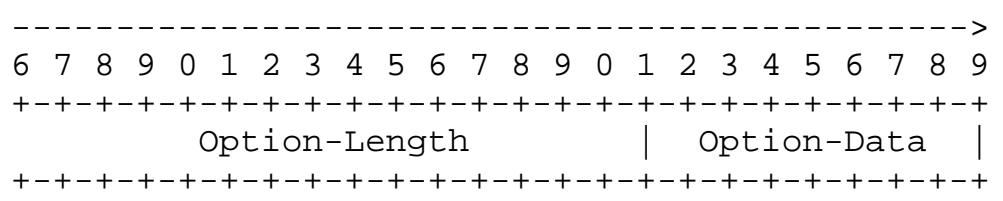

(2)

Address

This PPP-specified field is a single octet and contains the binary sequence 11111111 (hexadecimal 0xFF), the All-Stations address. PPP does not assign individual station addresses. The All-stations address must be recognized and received by all devices. For more information on this field, refer to "The Point-to-Point Protocol (PPP) for the Transmission of Multi-protocol Datagrams over Pointto-Point Links." (References, [1])

\section{Control}

This PPP-specified field is a single octet and contains the binary sequence 00000011 (hexadecimal 0x03), the Unnumbered Information (UI) command with the $\mathrm{P} / \mathrm{F}$ bit set to zero.

For more information on this field, refer to "The Point-to-Point Protocol (PPP) for the Transmission of Multi-protocol Datagrams over Point-to Point Links." (References, [1])

Note: Hereafter the Address and Control fields will be represented together as a 2-octet field containing "OxFF03".

Protocol-Type

The Protocol-Type field is two octets and contains the IETF-assigned protocol type value. Valid LAN extension interface protocol type values follow:

* 0x8041 (for control packets)

* 0x0041 (for data packets) 
Because the remote command options packet encapsulates LAN extension interface control data, the valid value for this field is 0x8041.

Code

The Code field is one octet and identifies the type of PPP-LEX packet. Valid values are as follows:

* 0x40 - LEX_RCMD_REQUEST packet

* 0x41 - LEX_RCMD_ACK packet

* 0x42 - LEX_RCMD_NAK packet

* 0x43 - LEX_RCMD_REJ packet

The host router sends the LEX_RCMD_REQUEST packet, and the LAN extension interface unit sends the LEX_RCMD_ACK, LEX_RCMD_NAK, and LEX_RCMD_REJ packets.

Identifier

The Identifier field is one octet and contains a randomly generated value. The value aids matching requests and replies. It is recommended that a non-zero value be used for the identifier. That is, zero could be used in the future for unsolicited messages from the LAN extension interface unit. Valid values are 0x01-0xFF.

Length

The Length field is two octets and indicates the length in octets of the entire packet, including the Code, Identifier, Length, and remote command options fields.

Option-Type

The Option-Type field is one octet and identifies the remote command option being transmitted. Valid values are as follows:

* 0x01 - Filter Protocol Type

* 0x02 - Filter MAC Address

* $0 \times 03$ - Set Priority

* 0x04 - Disable LAN Extension Ethernet Interface

* 0x05 - Enable LAN Extension Ethernet Interface

* 0x06 - Reboot LAN Extension Interface Unit

* 0x07 - Request Statistics

* 0x08 - Download Request

* 0x09 - Download Data

* 0x0A - Download Status

* 0x0B- Inventory Request 
Each remote command option is discussed in detail in its own section later in this document.

Option-Flags

This field is one octet and further specifies the remote command option, containing specific actions that must be followed.

Option-Length

The Option-Length field is two octets and specifies the length in octets of the remote command option fields, including the OptionType, Option-Flags, Option-Length, and Option-Data fields.

Option-Data

Option-Data field contains data relating to the remote command option specified in the Option-Type field.

\subsection{Conditions for sending PPP-LEX Packet}

This section describes the general conditions under which PPP-LEX packet types are sent. For specific information by remote command, refer to the appropriate remote command section later in this document.

LEX RCMD_REQUEST Packet

The host router sends LEX_RCMD_REQUEST packets to the LAN extension interface unit to initiate a remote command request. Until the host router receives a LEX_RCMD_ACK, LEX_RCMD_NAK, or LEX_RCMD_REJ packet from the LAN extension interface unit, the host router continues to send the LEX_RCMD_REQUEST packet a default number of times, at which point the host router times out.

LEX_RCMD_ACK Packet

The LAN extension interface unit responds to a LEX_RCMD_REQUEST packet with a LEX_RCMD_ACK packet when it correctly receives the request and is able to perform the request.

LEX RCMD_NAK Packet

The LAN extension interface unit responds to a LEX_RCMD_REQUEST packet with a LEX_RCMD_NAK packet when the LAN extension interface unit recognizes all the elements of the remote command option, but some elements are not acceptable. Upon receipt of a LEX_RCMD_NAK packet, the host router immediately stops sending the request. 
LEX RCMD_REJ Packet

The LAN extension interface unit responds to a LEX_RCMD_REQUEST packet with a LEX_RCMD_REJ packet when the Option-Type value in the request packet is invalid. Invalid Option-Type values are those less than $0 x 01$ or greater than 0x0B. Currently, this is the only condition under which the LAN extension interface unit sends a LEX_RCMD_REJ packet. Upon receipt of a LEX_RCMD_REJ packet, the host router immediately stops sending the request.

The following sections detail each of the 11 remote command options. The sections provide a general description of the option and then specify the option's Option-Type, Option-Flags, Option-Length, and Option-Data fields. In addition, the sections describe the return messages from the LAN extension interface unit.

\subsection{Filter Protocol Type}

The host router sends a LEX_RCMD_REQUEST packet with an Option-Type of $0 \times 01$ to the LAN extension interface unit to configure the LAN extension interface unit to filter inbound packets by protocol type. A protocol type filter determines whether or not the LAN extension interface unit forwards packets of a specific protocol type to the host router. A protocol type filter consists of a 16-bit value, 16bit mask, and a permit or deny field. (See the "Option-Data Field Descriptions" section for more information on these filter fields.)

A LEX_RCMD_REQUEST packet can contain 0 to 200 (depending on MTU size) protocol type filters. When a LEX_RCMD_REQUEST packet contains multiple filters, they are concatenated.

The LAN extension interface unit applies the protocol type filters to each inbound packet's protocol type field in the order in which the filters exist in the filter table. A packet must be permitted by one of the filters before the LAN extension interface unit can forward the packet across the serial link.

The following example is a filtering algorithm:

if (protocol_type_field \& ( filter_mask)) == filter_value) if (permit/deny_field == PERMIT) <forward packet on serial LAN> else <DROP PACKET>

The protocol type filter should also be applied to the DIX type code field of Ethernet II frames as well as to IEEE 802.2 SNAP packets. 


\subsection{LEX RCMD_REQUEST-Filter Protocol Type}

Figure 6 shows a frame format summary of a LEX_RCMD_REQUEST packet for the Filter Protocol Type remote command option. The host router transmits the fields from left to right.

Figure 6 LEX_RCMD_REQUEST Packet Frame Format - Filter Protocol Type PPP Header

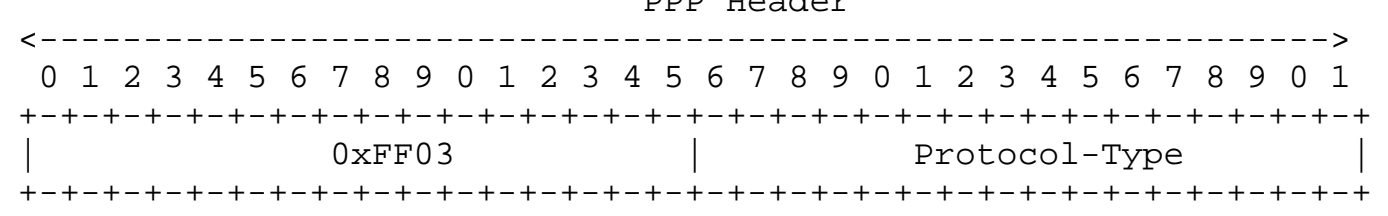

(2 octets)

LAN Extension Interface Protocol Header

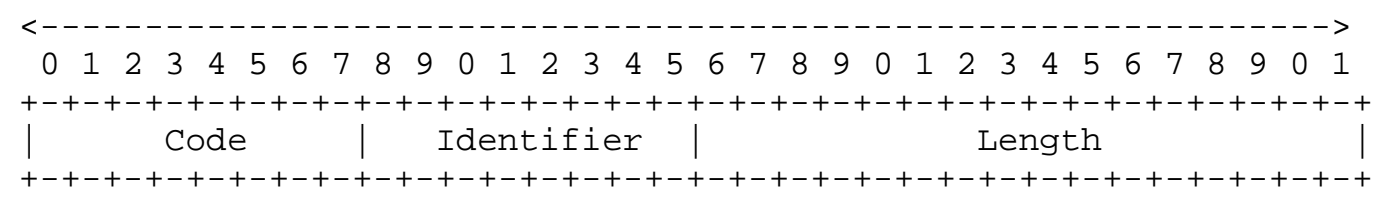

LAN Extension Interface Remote Command Options

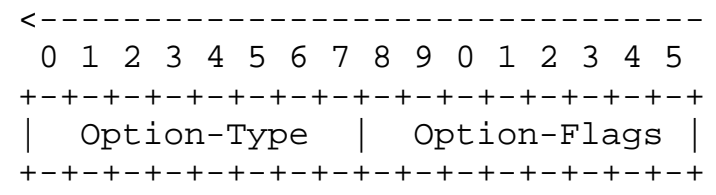

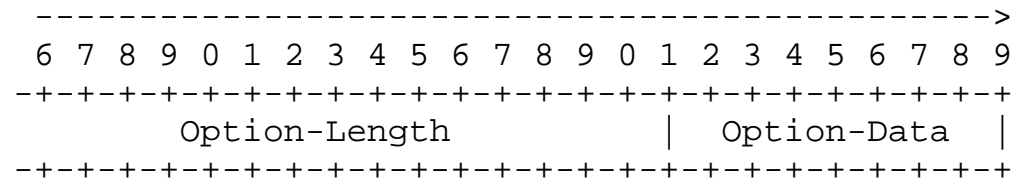

(2)

Where the Option-Data field contains the following fields:

$\begin{array}{llllllllllllllllllllllllllllllll}0 & 1 & 2 & 3 & 4 & 5 & 6 & 7 & 8 & 9 & 0 & 1 & 2 & 3 & 4 & 5 & 6 & 7 & 8 & 9 & 0 & 1 & 2 & 3 & 4 & 5 & 6 & 7 & 8 & 9 & 0 & 1\end{array}$

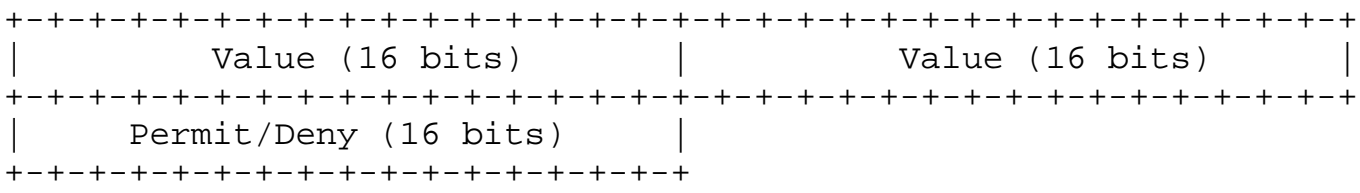




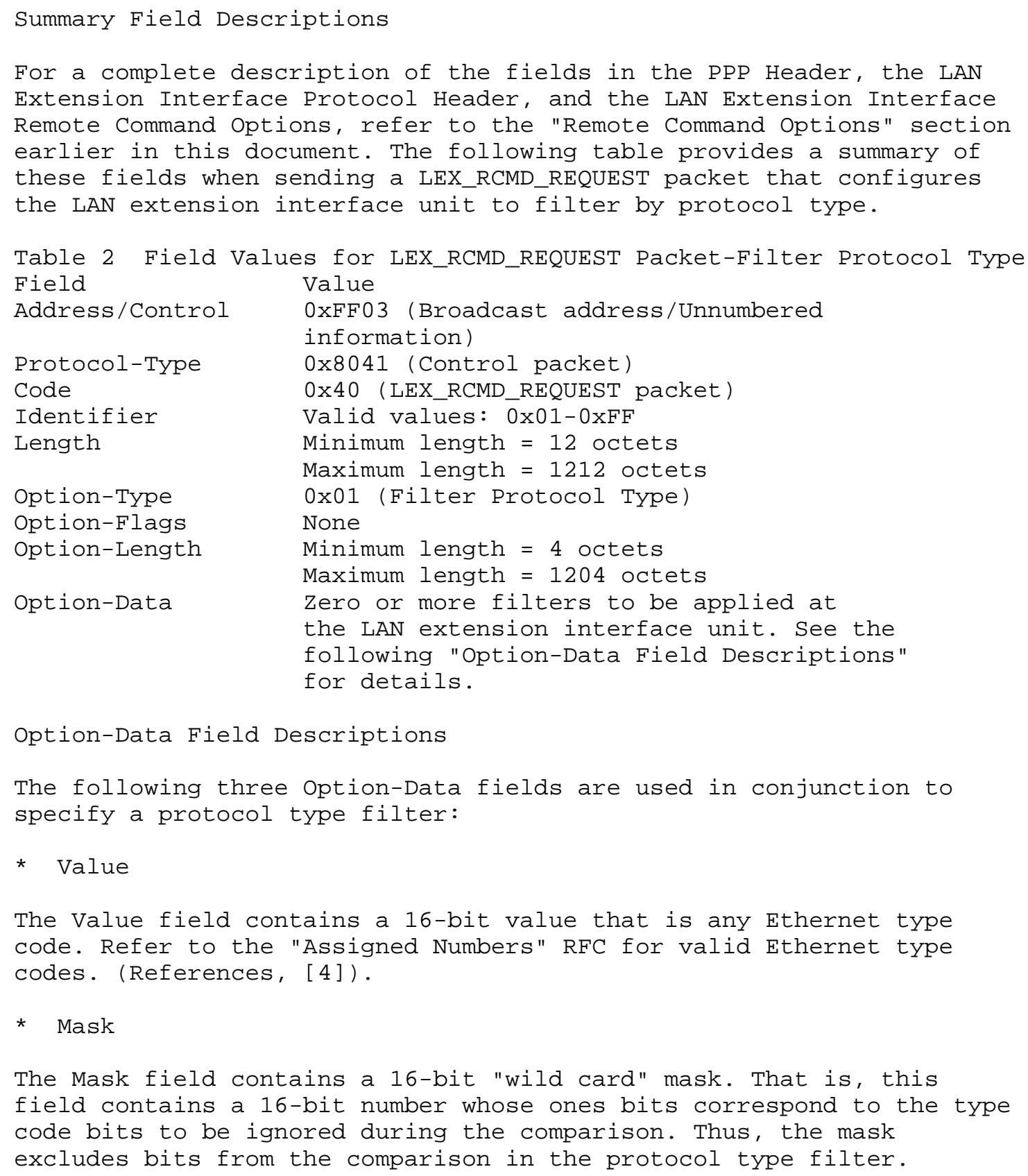


* Permit/Deny

The Permit/Deny field determines whether a protocol type filter permits or denies inbound frames to pass to the host router. A permit value is a non-zero value that allows frames of a specific protocol type to pass to the host router. A deny value is a zero value that does not allow frames of a specific protocol type to pass to the host router.

Implementation Notes

Each LEX_RCMD_REQUEST packet is a complete specification of all protocol type filters and replaces any previously established filters.

Note the following special cases:

* A LEX_RCMD_REQUEST packet with an Option-Length field equal to four (without any filter entries) instructs the LAN extension interface unit to turn off protocol type filtering. All MAC protocol types are forwarded.

* A LEX_RCMD_REQUEST packet with a filter entry of 0x0000 in the Value field, $0 x F F F$ in the Mask field, and a positive value in the Permit/Deny field means that if previous filters in the filter list do not permit the inbound packet then this filter entry will. This filter entry is typically the last filter in a list of filters contained within the Option-Data field.

* A LEX_RCMD_REQUEST packet with a filter entry of 0x0000 in the Value field, OxFFFF in the Mask field, and a zero in the Permit/Deny field, means that the LAN extension interface unit must deny all protocol types. This filter is typically the last filter in a list of filters contained within the Option-Data field.

\subsection{Response Packets - Filter Protocol Type}

The following packets are valid responses to the Filter Protocol Type LEX_RCMD_REQUEST packet:

* LEX_RCMD_ACK - Filter Protocol Type

The LAN extension interface unit sends a LEX_RCMD_ACK packet in response to the Filter Protocol Type LEX_RCMD_REQUEST packet when the LAN extension interface unit correctly receives the Filter Protocol Type remote command option and applies all filter entries to its filter table. All filter entries are returned to the host router in the LEX_RCMD_ACK packet. 
* LEX_RCMD_NAK - Filter Protocol Type

The LAN extension interface unit sends a LEX_RCMD_NAK packet in response to the Filter Protocol Type LEX_RCMD_REQUEST packet when the request contains an incorrect number of bytes in the filter or when there are no more filter entries available. The LAN extension interface unit continues to use the previous filter table (that is, the filter table that existed prior to the receipt of the request). The host router should signal an error to the user/network administrator. All filter entries are returned to the host router in the LEX_RCMD_NAK packet.

* LEX_RCMD-REJ - Filter Protocol Type

See the "Conditions for sending PPP-LEX Packets" section earlier in this document for more information on this packet type.

Table 3 summarizes the field values of Filter Protocol Type LEX_RCMD_ACK, LEX_RCMD_NAK, and LEX_RCMD_REJ packets.

Table 3 Field Values for Response Packets - Filter Protocol Type

$\begin{array}{ll}\text { Field } & \text { Value } \\ \text { Address/Control } & \text { OxFF03 (Broadcast address/Unnumbere } \\ & \text { information) } \\ \text { Protocol-Type } & \text { Ox804 (Control packet) } \\ \text { Code } & \text { Valid values: } \\ & \star 0 \times 41 \text { (LEX_RCMD_ACK packet) } \\ & \star 0 \times 42 \text { (LEX_RCMD_NAK packet) } \\ & \star 0 \times 43 \text { (LEX_RCMD_REJ packet) } \\ & \text { The same value as that sent by the } \\ \text { Identifier } & \text { LEX_RCMD_REQUEST packet } \\ \text { Length } & \text { The same value as that sent by the } \\ & \text { LEX_RCMD_REQUEST packet } \\ \text { Option-Type } & \text { Ox01 (Filter Protocol Type) } \\ \text { Option-Flags } & \text { None } \\ \text { Option-Length } & \text { The same value as that sent by the } \\ \text { Option-Data } & \text { LEX_RCMD_REQUEST packet } \\ & \text { The filter entries sent in the } \\ & \text { LEX_RCMD_REQUEST packet }\end{array}$

\subsection{Filter MAC Address}

The host router sends a LEX_RCMD_REQUEST packet with an Option-Type of 0x02 to the LAN extension interface unit to configure the LAN extension interface unit to filter inbound packets by source MAC address. A MAC address filter determines whether or not the LAN extension interface unit forwards packets with a specific source MAC 
LAN Extension Interface Remote Command Options

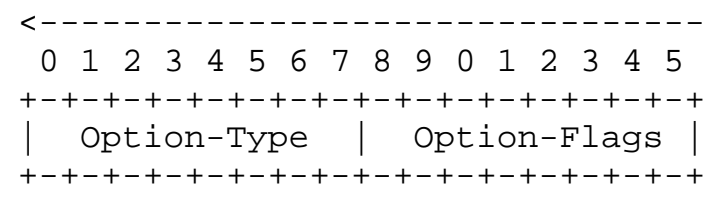

(1)

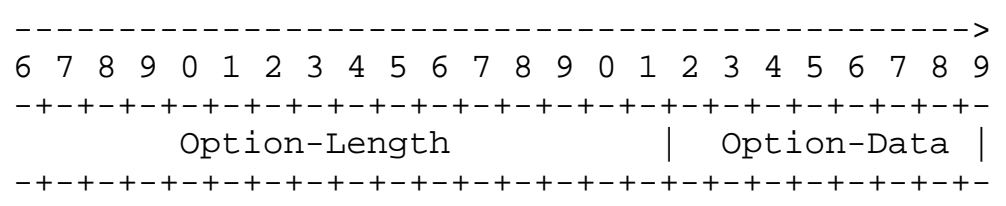

(2)

Where the Option-Data field contains the following fields:

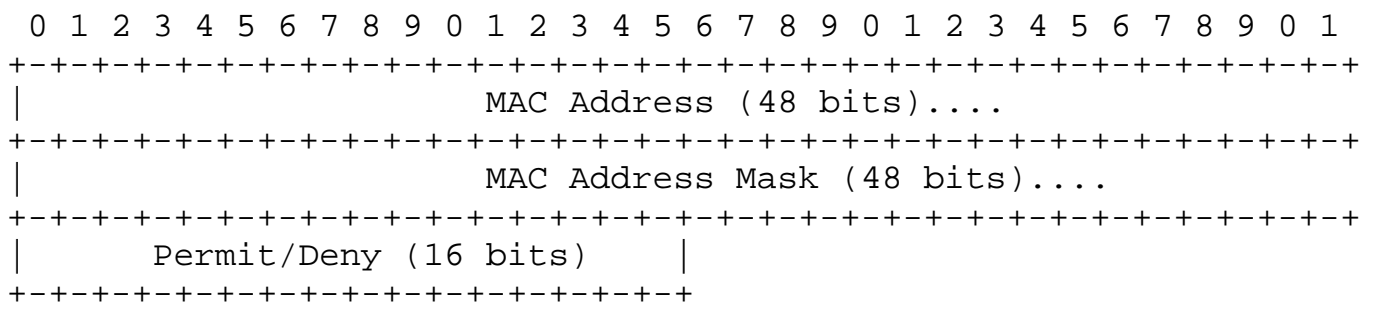

Summary Field Descriptions

For a complete description of the fields in the PPP Header, the LAN Extension Interface Protocol Header, and the LAN Extension Interface Remote Command Options, refer to the "Remote Command Options" section earlier in this document. Table 4 provides a summary of these fields when sending a LEX_RCMD_REQUEST packet that configures the LAN extension interface unit to filter by source MAC address.

Table 4 Field Values for LEX_RCMD_REQUEST Packet - Filter MAC Address

$\begin{array}{ll}\text { Field } & \text { Value } \\ \text { Address/Control } & \text { OxFF03 (Broadcast address/Unnumbered } \\ & \text { information) } \\ \text { Protocol-Type } & \text { Ox804 (Control packet) } \\ \text { Code } & \text { Valid (LEX_RCMD_REQUEST packet) } \\ \text { Identifier } & \text { Minimum length }=12 \text { octets } \\ \text { Length } & \text { Maximum length }=1412 \text { octets } \\ & \text { Ox02 (Filter MAC Address) } \\ \text { Option-Type } & \text { None } \\ \text { Option-Flags } & \text { Minimum length }=4 \text { octets } \\ \text { Option-Length } & \end{array}$




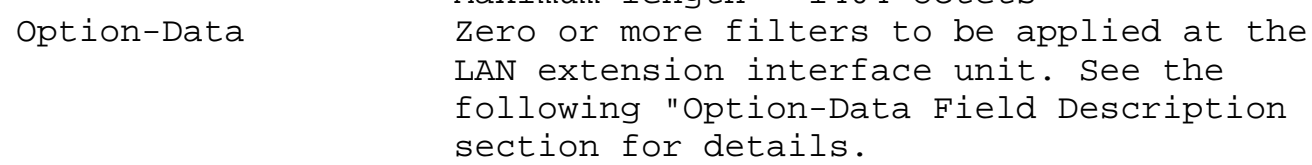

The Permit/Deny field determines whether or not a MAC address filter permits or denies inbound frames of a specific MAC address to pass to the host router. A permit value is a non-zero value that allows frames of a specific MAC address to pass to the host router. A deny value is a zero value that does not allow frames of a specific MAC address to pass to the host router.

Implementation Notes

Each LEX_RCMD_REQUEST packet is a complete specification of all MAC address filters and replaces any previously established filters.

A LEX_RCMD_REQUEST packet with an Option-Length field equal to four (without any filter entries) instructs the LAN extension interface unit to turn off filtering. All MAC addresses, except local destination addresses cached in the self-learning filter, are forwarded.

4.2 Response Packets - Filter MAC Address

The following packets are valid responses to the Filter MAC Address LEX_RCMD_REQUEST packet: 
* LEX_RCMD_ACK - Filter MAC Address

The LAN extension interface unit sends a LEX_RCMD_ACK packet in response to a Filter MAC Address LEX_RCMD_REQUEST packet when the LAN extension interface unit correctly receives the Filter MAC Address remote command option and applies the entries to its filter table. All MAC address filter entries are returned in the LEX_RCMD_ACK packet.

* LEX_RCMD_NAK - Filter MAC Address

The LAN extension interface unit sends a LEX_RCMD_NAK packet in response to the Filter MAC Address LEX_RCMD_REQUEST packet when the request contains an incorrect number of bytes in the filter or when there are no more filter entries available. The LAN extension interface unit continues to use the previous filter table (that is, the filter table that existed prior to the receipt of the request). The host router should signal an error to the user/network administrator. All filter entries are returned in the LEX_RCMD_NAK packet.

* LEX_RCMD-REJ - Filter MAC Address

See the "Conditions for Sending PPP-LEX Packets" section earlier in this document for more information on this packet type.

Table 5 summarizes the field values of Filter MAC Address LEX_RCMD_ACK, LEX_RCMD_NAK, and LEX_RCMD_REJ packets.

Table 5 Field Values for Response Packets - Filter MAC Address

$\begin{array}{ll}\text { Field } & \text { Value } \\ \text { Address/Control } & \text { OxFFO3 (Broadcast address/Unnumbered } \\ & \text { information) } \\ \text { Protocol-Type } & \text { 0x8041 (Control packet) } \\ \text { Code } & \text { Valid values: } \\ & \star 0 \times 41 \text { (LEX_RCMD_ACK packet) } \\ & \star 0 \times 42 \text { (LEX_RCMD_NAK packet) } \\ & \star 0 x 43 \text { (LEX_RCMD_REJ packet) } \\ & \text { The same value as that sent by the } \\ \text { Identifier } & \text { LEX_RCMD_REQUEST packet } \\ \text { Length } & \text { The same value as that sent by the } \\ & \text { LEX_RCMD_REQUEST packet } \\ \text { Option-Type } & \text { Ox02 (Filter MAC Address) } \\ \text { Option-Flags } & \text { None } \\ \text { Option-Length } & \text { The same value as that sent by the } \\ \text { Option-Data } & \text { LEX_RCMD_REQUEST packet } \\ & \text { The filter entries sent in the }\end{array}$




\section{0 set Priority}

The host router sends a LEX_RCMD_REQUEST with an Option-Type of 0x03 to the LAN extension interface unit to establish the sending priority of different protocol type packets from the LAN extension interface unit to host router. There are four levels of priority:

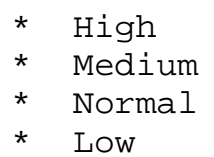

Packets are classified according to protocol type and then are queued to one of four output queues on the LAN extension interface unit that correspond to the above priority levels. When the LAN extension interface unit is ready to transmit a packet, it scans the priority queues in order, from the highest to lowest, to find the highest priority packet.

\subsection{LEX RCMD_REQUEST - Set Priority}

To establish priority queues for each protocol type, the host router sends a set Priority LEX_RCMD_REQUEST packet. Figure 8 shows a frame format summary of such a LEX_RCMD_REQUEST packet. The host router transmits the fields from left to right.

Figure 8 LEX_RCMD_REQUEST Packet Frame Format - Set Priority

\section{PPP Header}

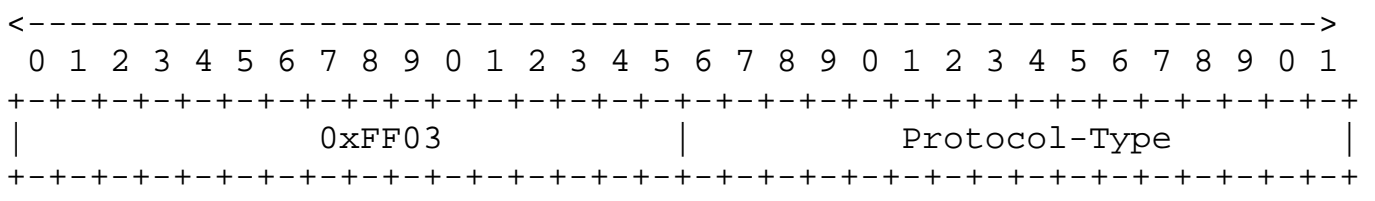

(2 octets)

LAN Extension Interface Protocol Header

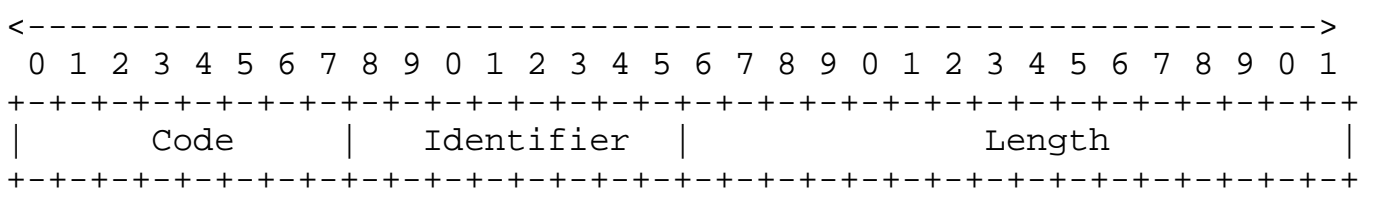

(1)

(1)
(2) 
LAN Extension Interface Remote Command Options

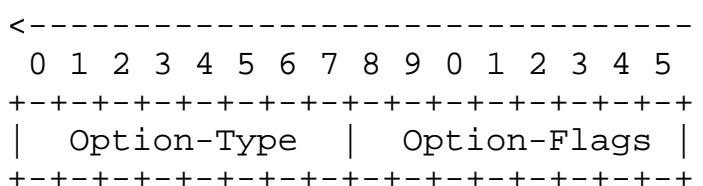

(1)

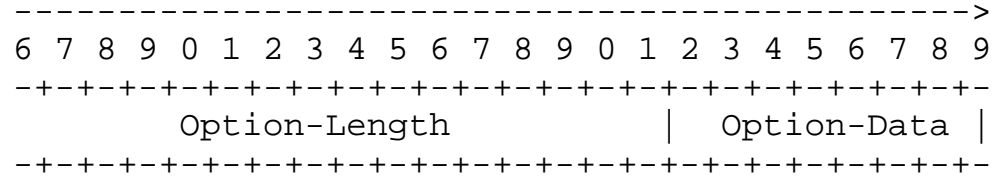

Where the option-Data field contains the following fields:

$\begin{array}{lllllllllllllllllllllllllllllllll}0 & 1 & 2 & 3 & 4 & 5 & 6 & 7 & 8 & 9 & 0 & 1 & 2 & 3 & 4 & 5 & 6 & 7 & 8 & 9 & 0 & 1 & 2 & 3 & 4 & 5 & 6 & 7 & 8 & 9 & 0 & 1\end{array}$ +-+-+-+-+-+-+-+-+-+-+-+-+-+-+-+-+-+-+-+-+-+-+-+-+-+-+-+-+-+-+-+-+ | Protocol Type (16 bits) | Protocol Value (16 bits) |

Summary Field Descriptions

For a complete descriptions of the fields in the PPP Header, the LAN Extension Interface Protocol Header, and the LAN Extension Interface Remote Command Options, refer to the "Remote Command Options" section earlier in this document. The following table provides a summary of these fields when sending a LEX_RCMD_REQUEST packet that sets priority queuing.

Table 6 Field Values for LEX_RCMD_REQUEST Packet - Set Priority

Field
Address/Control
Protocol-Type
Code
Identifier
Length
Option-Type
Option-Flags
Option-Length
Option-Data

Chapman, et al
Value

0xFF03 (Broadcast address/Unnumbered information)

0x8041 (Control packet)

0x40 (LEX_RCMD_REQUEST packet)

Valid values: 0x01-0xFF

Minimum length $=12$ octets

Maximum length $=1028$ octets

0x03 (Set Priority)

None

Minimum length $=4$ octets

Maximum length $=1020$ octets

Protocol Type and Priority Value. See the following "Option-Data Field Description" section for details. 
Option-Data Field Descriptions

The following option-Data fields set the priority queuing of different protocol type packets.

* Protocol Type

The Protocol Type field contains a 16-bit number that is any Ethernet type code. See the most recent "Assigned Numbers" RFC for the correct Ethernet type code.

* Priority Value

The Priority Value field specifies the priority queue for the protocol type specified in the Protocol Type field. Valid values are as follows:

- 0 - High priority queue

- 1 - Medium priority queue

- 2 - Normal priority queue

- 3 - Low priority queue

Sending a LEX_RCMD_REQUEST packet with an Option-Length of four (no priority entries) disables priority queuing. When disabled, the LAN extension interface unit transfers all packets at a normal (2)

priority level. When a new priority is specified, it overwrites the previous setting.

5.2 Response Packets - set Priority

The following packets are valid responses to the set Priority

LEX_RCMD_REQUEST packet.

* LEX_RCMD_ACK - Set Priority

See the "Conditions for Sending PPP-LEX Packets" section earlier in this document for more information on this packet type.

* LEX_RCMD_NAK - Set Priority

The LAN extension interface unit sends a LEX_RCMD_NAK packet in response to the set Priority LEX_RCMD_REQUEST packet when the request contains an incorrect number of bytes in the message, when necessary resources are not available, or when the specified priority is invalid. 
* LEX_RCMD-REJ - Set Priority

See the "Conditions for Sending PPP-LEX Packets" section earlier in this document for more information on this packet type. Table 7 summarizes the field values of set Priority LEX_RCMD_ACK, LEX_RCMD_NAK, and LEX_RCMD_REJ packets.

\section{Table 7 Field Values for Response Packets - Set Priority}

Field
Address/Contro
Protocol-Type
Code
Identifier
Length
Option-Type
Option-Flags
Option-Length
Option-Data

Value

0xFF03 (Broadcast address/Unnumbered information)

0x8041 (Control packet)

Valid values:

* 0x41 (LEX_RCMD_ACK packet)

* 0x42 (LEX_RCMD_NAK packet)

* 0x43 (LEX_RCMD_REJ packet)

A randomly generated value that aids in matching requests with replies

Minimum length $=12$ octets

Maximum length $=1028$ octets

0x03 (Set Priority)

None

Minimum length $=4$ octets

Maximum length $=1020$ octets

Protocol Type and Priority Value sent in the LEX_RCMD_REQUEST packet

6.0 Disable LAN Extension Ethernet Interface

The host router sends a LEX_RCMD_REQUEST with an Option-Type of 0x04 to the LAN extension interface unit to disable the LAN extension Ethernet interface. This remote command option stops data traffic from the LAN extension interface unit to the host router for troubleshooting or for reconfiguring the LAN extension interface unit. This remote command option only affects data traffic. PPP-LEX control packets can still be transferred over the serial link. 
6.1 LEX RCMD_REQUEST - Disable LAN Extension Ethernet Interface

Figure 9 shows a frame format summary of a LEX_RCMD_REQUEST packet for the Disable LAN Extension Ethernet Interface remote command option. The host router transmits the fields from left to right.

Figure 9 LEX_RCMD_REQUEST Packet Frame Format Disable LAN Extension Ethernet Interface

PPP Header

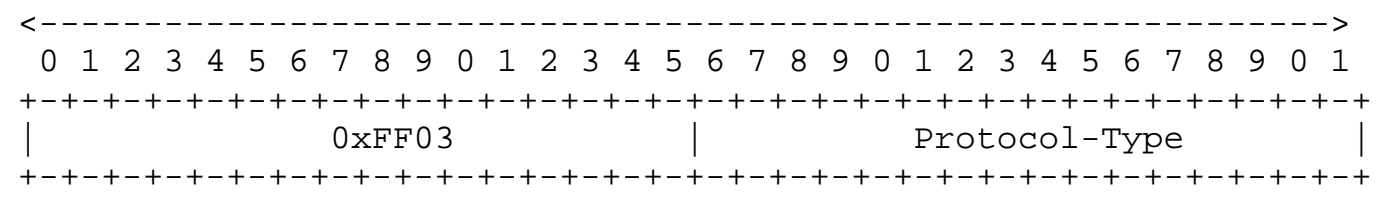

(2 octets)

LAN Extension Interface Protocol Header

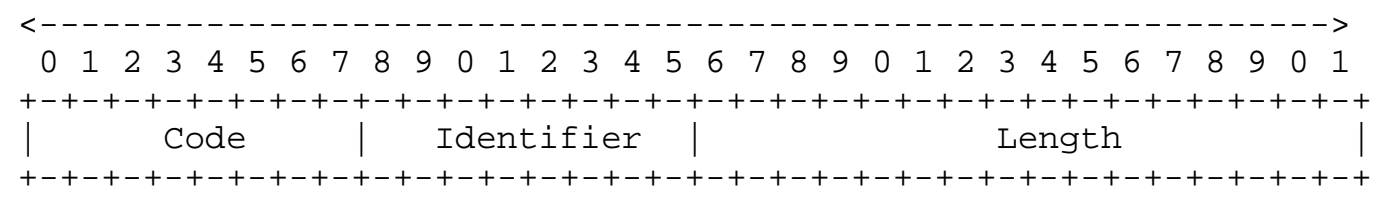

(1)

LAN Extension Interface Remote Command Options $<----------------------------------------------------------->$

$\begin{array}{llllllllllllllllllllllllllllllll}0 & 1 & 2 & 3 & 4 & 5 & 6 & 7 & 8 & 9 & 0 & 1 & 2 & 3 & 4 & 5 & 6 & 7 & 8 & 9 & 0 & 1 & 2 & 3 & 4 & 5 & 6 & 7 & 8 & 9 & 0 & 1\end{array}$ $+-+-+-+-+-+-+-+-+-+-+-+-+-+-+-+-+-+-+-+-+-+-+-+-+-+-+-+-+-+-+-+-+$ | Option-Type | Option-Flags | Option-Length

(1)

Summary Field Descriptions

For complete descriptions of the fields in the PPP Header, the LAN Extension Interface Protocol Header, and the LAN Extension Interface Remote Command Option, refer to the "Remote Command Options" section earlier in this document. The following table provides a summary of these fields when sending a LEX_RCMD_REQUEST packet that disables the LAN extension interface unit. 
Table 8 Field Values for LEX_RCMD_REQUEST Packet Disable LAN Extension Ethernet Interface

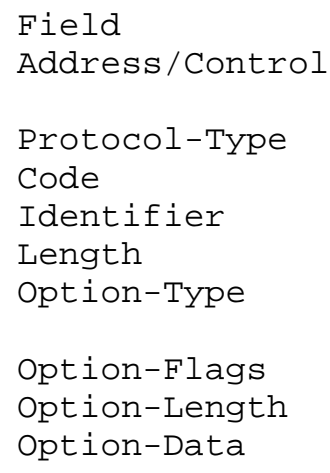




\subsection{Enable LAN Extension Ethernet Interface}

The host router sends a LEX_RCMD_REQUEST with an Option-Type of 0x05 to the LAN extension interface unit to enable the LAN extension Ethernet interface. This remote command option allows LAN traffic to flow into the LAN extension interface unit after the interface has been disabled.

\subsection{LEX RCMD_REQUEST - Enable LAN Extension Ethernet Interface}

Figure 10 shows a frame format summary of a LEX_RCMD_REQUEST packet for an Enable LAN Extension Ethernet Interface remote command option. The host router transmits the fields from left to right.

Figure 10 LEX_RCMD_REQUEST Packet Frame Format - Enable LAN Extension Ethernet Interface

\section{PPP Header}

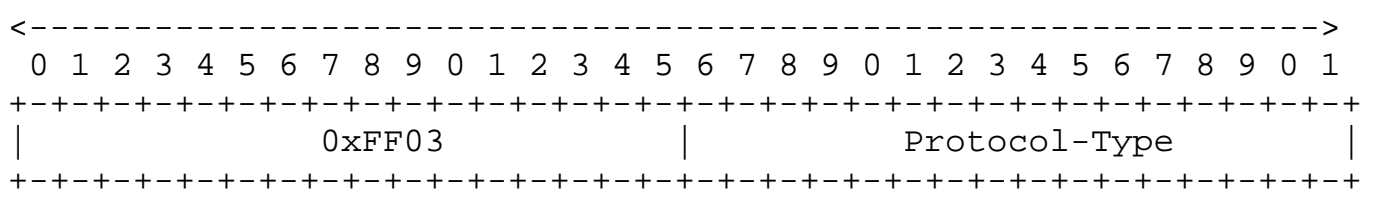

(2 octets)

LAN Extension Interface Protocol Header

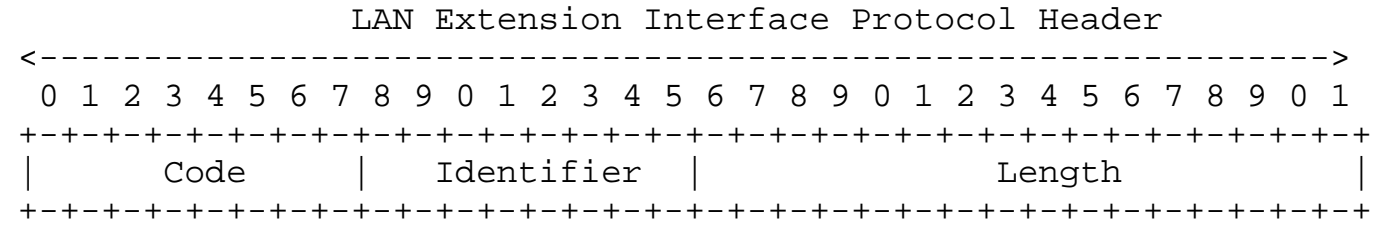

(1)
(2)

LAN Extension Interface Remote Command Options

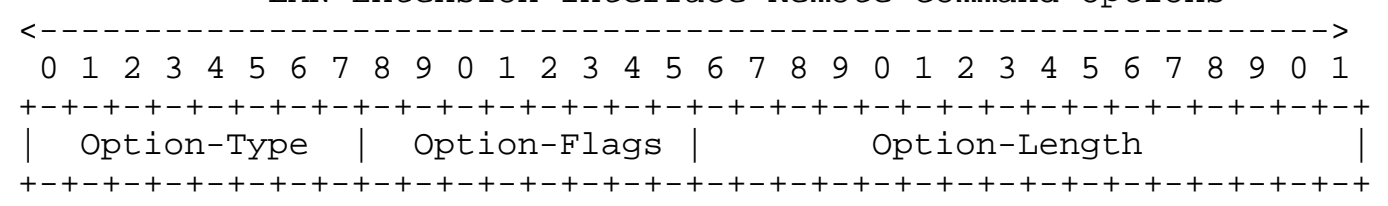

(1)
(2)

Summary Field Descriptions

For complete descriptions of the fields in the PPP Header, the LAN Extension Interface Protocol Header, and the LAN Extension Interface Remote Command Option, refer to the "Remote Command Options" section earlier in this document. Table 10 provides a summary of these fields when sending a LEX_RCMD_REQUEST packet that enables the LAN extension 
$\begin{array}{ll}\text { Option-Flags } & \text { None } \\ \text { Option-Length } & 4 \text { octets } \\ \text { Option-Data } & \text { None }\end{array}$

8.0 Reboot LAN Extension Interface Unit

The host router sends a LEX_RCMD_REQUEST packet with an Option-Type of $0 \times 06$ to the LAN extension interface unit to cause the LAN extension interface unit to reboot itself. The Option-Flags field specifies the boot mode for the LAN extension interface unit. There are two boot modes:

* The first boot mode (Option-Flag 0x00) is the default. The default boot mode causes the LAN extension interface unit to check for a valid Flash image and to boot from it if it exists. If a valid Flash image does not exist, the default boot mode causes the LAN extension interface unit to boot from the PROM image. Similarly, if the Flash image is bad, then the LAN extension interface unit recovers by booting from the PROM image.

* The second boot mode (Option-Flag 0x01) forces the LAN extension interface unit to boot from the PROM image.

\subsection{LEX RCMD_REQUEST - Reboot LAN Extension Interface Unit}

Figure 11 shows a frame format summary of a LEX_RCMD_REQUEST packet for a Reboot LAN Extension Interface Unit remote command option. The host router transmits the fields from left to right.

Figure 11 LEX_RCMD_REQUEST Packet Frame Format Reboot LAN Extension Interface Unit

PPP Header

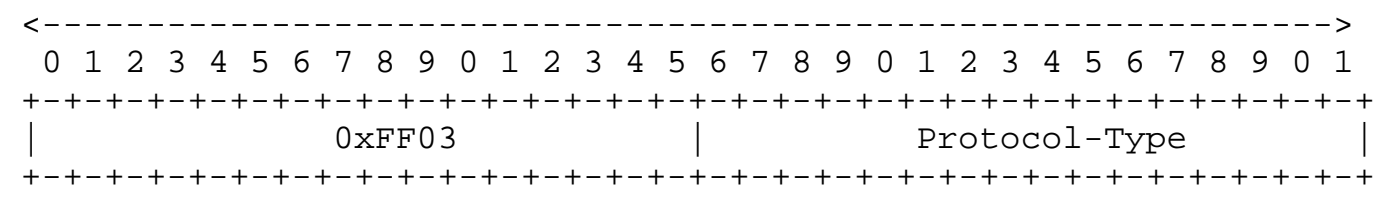

(2 octets)

LAN Extension Interface Protocol Header

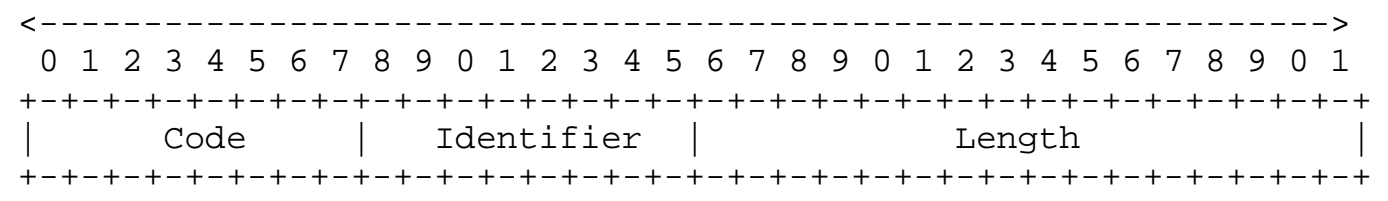

(2) 
LAN Extension Interface Remote Command Options

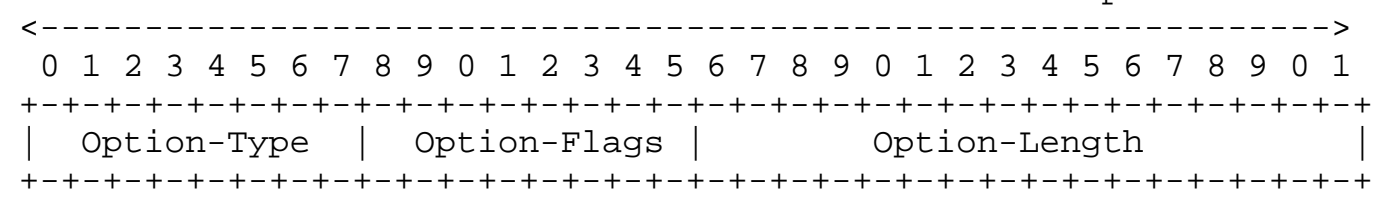

(1)

(2)

Summary Field Descriptions

For complete descriptions of the fields in the PPP Header, the LAN Extension Interface Protocol Header, and the LAN Extension Interface Remote Command Option, refer to the "Remote Command Options" section earlier in this document. Table 12 provides a summary of these fields when sending a LEX_RCMD_REQUEST packet that instructs the LAN extension interface unit to reboot.

Table 12 Field Values for LEX_RCMD_REQUEST Packet Reboot LAN Extension Interface Unit

Field

Address/Control

Protocol-Type

Code

Identifier

Length

Option-Type

Option-Flags

Option-Length

option-Data
Value

0xFF03 (Broadcast address/Unnumbered

information)

0x8041 (Control packet)

0x40 (LEX_RCMD_REQUEST packet)

Valid values: 0x01-0xFF

12 octets

0x06 (Reboot LAN Extension Interface Unit)

Valid values:

* 0x00 - Reboot from Flash image if it exists. If not, reboot from PROM. (This value does not force the LAN extension interface unit to reboot from PROM.)

* 0x01 - Reboot from PROM explicitly.

4 octets

None

\subsection{Response Packets - Reboot LAN Extension Interface Unit}

LEX_RCMD_ACK, LEX_RCMD_NAK, and LEX_RCMD-REJ packets are valid responses to the Reboot LAN Extension Interface Unit LEX_RCMD_REQUEST packet. Refer to the "Conditions for sending PPP-LEX Packets" section earlier in this document for more information on when the LAN extension interface unit sends each of these response packets. Note that the LAN extension interface unit reboots after sending the LEX_RCMD_ACK packet. 
The frame format for the response packets mirrors that of the request. Table 13 summarizes the field values for Reboot LAN Extension Interface Unit LEX_RCMD_ACK, LEX_RCMD_NAK, and LEX_RCMD_REJ packets.

Table 13 Field Values for Response Packets Reboot LAN Extension Interface Unit

\author{
Field \\ Address/Control \\ Protocol-Type \\ Code
}

Identifier

Length

Option-Type

Option-Flags

Option-Length

Option-Data

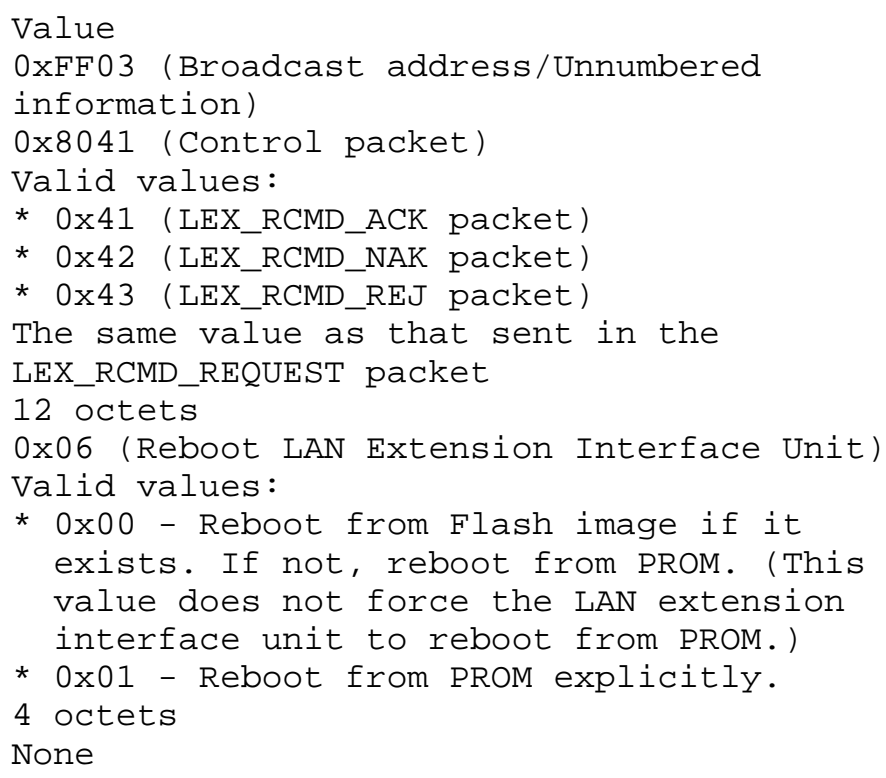

9.0 Request Statistics

The host router issues a LEX_RCMD_REQUEST packet with an Option-Type of 0x07 to obtain statistics information from the LAN extension interface unit. The host router may want statistics information about the following:

* Serial interface only

* LAN interface only

* Both the serial and LAN interfaces

The host router may also send a Statistics Request LEX_RCMD_REQUEST packet to reset statistics in the LAN extension interface unit.

9.1 LEX RCMD_REQUEST - Request Statistics

Figure 12 shows a summary frame format of a LEX_RCMD_REQUEST packet for a statistics Request remote command option. The host router transmits the fields from left to right. 
Figure 12 LEX_RCMD_REQUEST Packet Frame Format - Request Statistics PPP Header

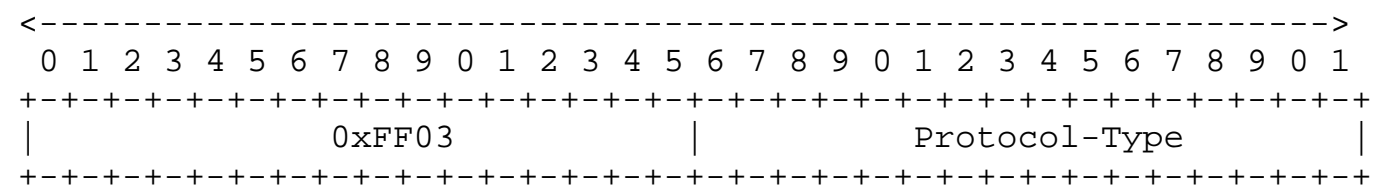

(2 octets)

LAN Extension Interface Protocol Header

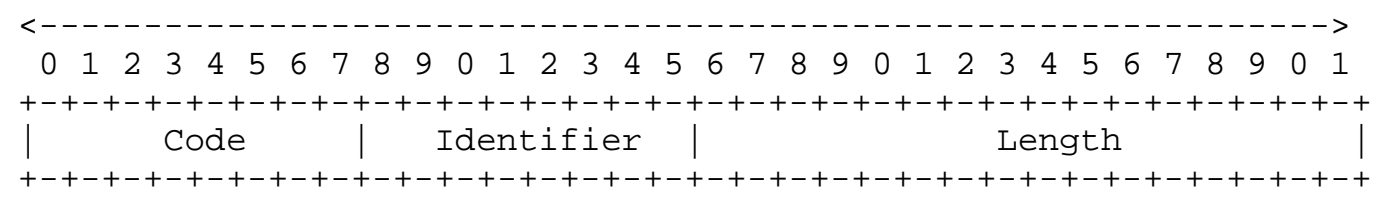

LAN Extension Interface Remote Command Option

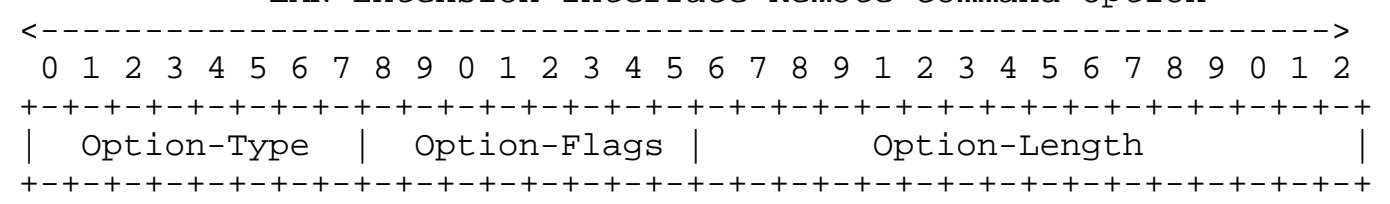

Summary Field Descriptions

For complete descriptions of the fields in the PPP Header, the LAN Extension Interface Protocol Header, and the LAN Extension Interface Remote Command Option, refer to the "Remote Command Options" section earlier in this document. Table 14 provides a summary of these fields when sending a LEX_RCMD_REQUEST packet that requests statistics of the LAN extension interface unit.

\section{Table 14 Field Values for LEX_RCMD_REQUEST Packet - Request statistics}

Field
Address/Control
Protocol-Type
Code
Identifier
Length
Option-Type
Option-Flags

Chapman, et al
Value

0xFF03 (Broadcast address/Unnumbered information)

0x8041 (Control packet)

0x40 (LEX_RCMD_REQUEST packet)

Valid values: $0 \times 01-0 \times F F$

12 octets

0x07 (Request statistics)

Valid values:

* 0x01 - Resets serial statistics in the LAN 
Option-Length Option-Data extension interface unit

* 0x02 - Returns serial statistics from the LAN extension interface unit

* 0x04 - Returns LAN statistics from the LAN extension interface unit

* 0x08 - Resets LAN statistics in the LAN

* extension interface unit

If both serial and LAN statistics are desired, the corresponding bits of this field should be set (that is, 0x06). The serial interface statistics appear in the response packet before the LAN statistics. 4 octets None

\subsection{LEX RCMD_ACK - Request Statistics}

The normal response to a Statistics Request LEX_RCMD_REQUEST packet is a LEX_RCMD_ACK packet. This acknowledgment packet has an optionType, an Option-Flags, and an Option-Length field followed by one or two blocks of statistics data. The value in the Option-Flags field indicates whether the packet has one or two blocks of statistics. For more information on these values, see the following "Summary Field Descriptions" section.

The frame format for the Statistics Request LEX_RCMD_ACK packet follows. The LAN extension interface unit transmits the fields from left to right.

Figure 13 LEX_RCMD_ACK Packet Frame Format - Request Statistics

PPP Header

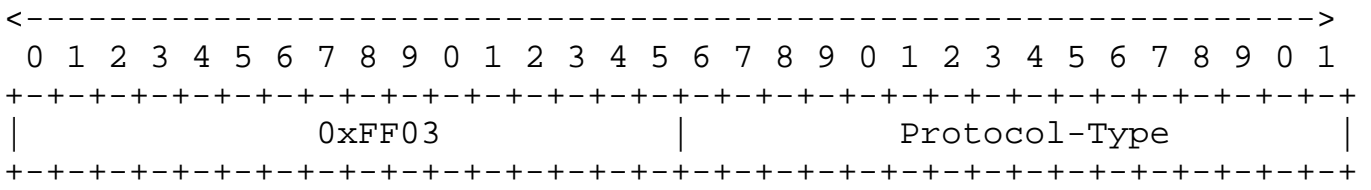

(2 octets)

(2)

LAN Extension Interface Protocol Header

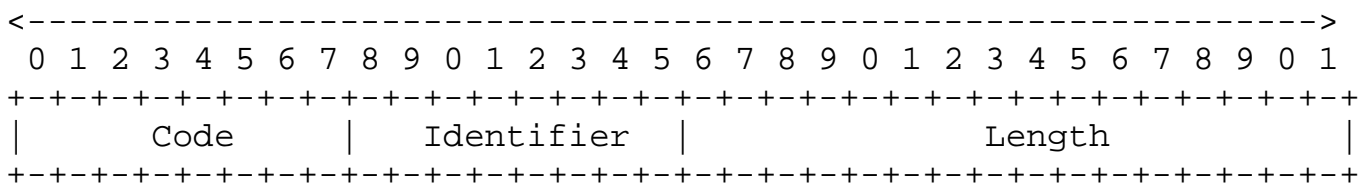


LAN Extension Interface Remote Command Options

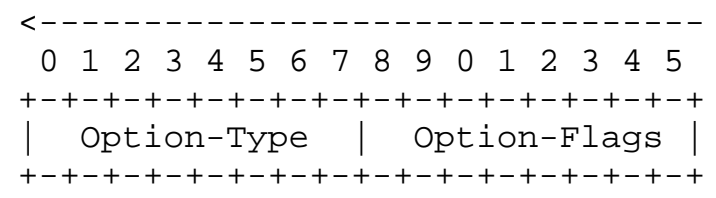

(1)

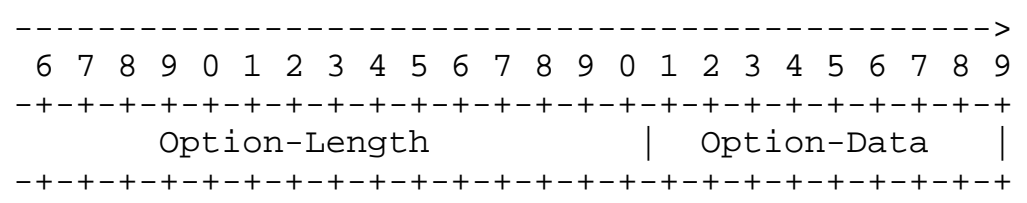

Where the Option-Data fields contains one or both of the following statistics blocks:

Serial Interface Statistics Block:

(32 bits)

Serial Line Input, Number of Packets Received
+-+-+-+-+-+-+-+-+-+-+-+-+-+++-+-+-+-+-+-+-+-+-+-+-+-+-+-+-+-+-+-+
Serial Line Input, Number of CRC Erros
+-+-+-+-+-+-+-+-+-+-+-+-+-+-+-+-+-+-+-+-+-+-+-+-+-+-+-+-+-+-+-+-+
Serial Line Input, Number of Framing Errors
+-+-+-+-+-+-+-+-+-+-+-+-+-+-+-+-+-+-+-+-+-+-+-+-+-+-+-+-+-+-+-+-+
Serial Line Input, Number of Overruns
+-+-+-+-+-+-+-+-+-+-+-+-+-+-+-+-+-+-+-+-+-+-+-+-+-+-+-+-+-+-+-+-+
Serial Line Input, Number of Packets Dropped
+-+-+-+-+-+-+-+-+-+-+-+-+-+-+-+-+-+-+-+-+-+-+-+-+-+-+-+-+-+-+-+-+
Serial Line Input, Number of Frame Aborts
+-+-+-+-+-+-+-+-+-+-+-+-+-+-+-+-+-+-+-+-+-+-+-+-+-+-+-+-+-+-+-+-+
Serial Line Input, Number of Packets in Error
+-+-+-+-+-+-+-+-+-+-+-+-+-+-+-+-+-+-+-+-+-+-+-+-+-+-+-+-+-+-+-+-+
Serial Line Input, Number of Packets Dropped Due to Lack of
Buffer Descriptors
+-+-+-+-+-+-+-+-+-+-+-+-+-+-+-+-+-+-+-+-+-+-+-+-+-+-+-+-+-+-+-+-+
Serial Line Input, Time of Last Packet Received
+-+-+-+-+-+-+-+-+-+-+-+-+-+-+-+-+-+-+-+-+-+-+-+-+-+-+-+-+-+-+-+-+
$\mid$ Serial Line Input, Average Data Rate
+-+-+-+-+-+-+-+-+-+-+-+-+-+-+-+-+-+-+-+-+-+-+-+-+-+-+-+-+-+-+-+-+
$\mid$ Serial Line Input, Average Packet Rate
+-+-+-+-+-+-+-+-+-+-+-+-+-+-+-+-+-+-+-+-+-+-+-+-+-+-+-+-+-+-+-+-+
$\mid$ Serial Line Input, Number of Octets Received
+-+-+-+-+-+-+-+-+-+-+-+-+-+-+-+-+-+-+-+-++-+-+-+-+-+-+-+-+-+-+-+
$\mid$ Serial Line Input, Number of Packets Dropped Due to No Buffers |




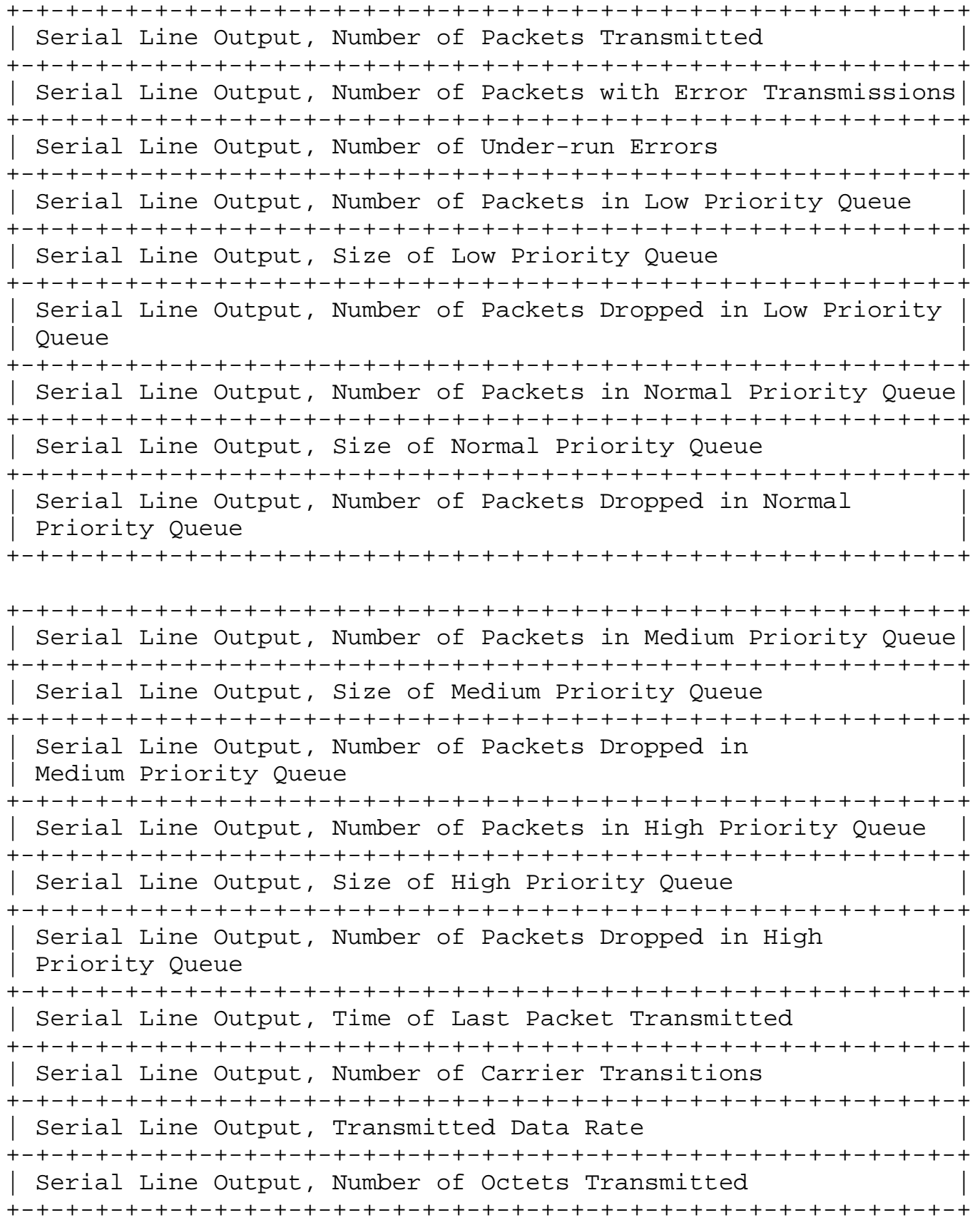


Lan Interface Statistics Block:

\section{(32 bits)}

| LAN Input, Number of Packets Received
+-+-+-+-+-+-+-+-+-+-+-+-+-+-+-+-+-+-+-+-+-+-+-+-+-+-+-+-+-+-+-+-+
$\mid$ LAN Input, Number of Packets Received with CRC Errors
+-+-+-+-+-+-+-+-+-+-+-+-+-+-+-+-+-+-+-+-+-+-+-+-+-+-+-+-+-+-+-+-+
LAN Input, Number of Packets Received with Framing Errors
+-+-+-+-+-+-+-+-+-+-+-+-+-+-+-+-+-+-+-+-+-+-+-+-+-+-+-+-+-+-+-+-+
LAN Input, Number of Overruns
+-+-+-+-+-+-+-+-+-+-+-+-+-+-+-+-+-+-+-+-+-+-+-+-+-+-+-+-+-+-+-+-+
LAN Input, Number of Packets Dropped Due to Congestion
+-+-+-+-+-+-+-+-+-+-+-+-+-+-+-+-+-+-+-+-+-+-+-+-+-+-+-+-+-+-+-+-+
LAN Input, Number of Frame Aborts
+-+-+-+-+-+-+-+-+-+-+-+-+-+-+-+-+-+-+-+-+-+-+-+-+-+-+-+-+-+-+-+-+
LAN Input, Number of Broadcast Packets Received
+-+-+-+-+-+-+-+-+-+-+-+-+-+-+-+-+-+-+-+-+-+-+-+-+-+-+-+-+-+-+-+-+
LAN Input, Number of Packets Received with Errors
+-+-+-+-+-+-+-+-+-+-+-+-+-+-+-+-+-+-+-+-+-+-+-+-+-+-+-+-+-+-+-+-+
LAN Input, Number of Packets Dropped Due to Lack of Buffer
Descriptors
+-+-+-+-+-+-+-+-+-+-+-+-+-+-+-+-+-+-+-+-+-+-+-+-+-+-+-+-+-+-+-+-+
$\mid$ LAN Input, Number of Runts (Too Small Packets) Received
+-+-+-+-+-+-+-+-+-+-+-+-+-+-+-+-+-+-+-+-+-+-+-+-+-+-+-+-+-+-+-+-+
LAN Input, Time of Last Packet Received
+-+-+-+-+-+-+-+-+-+-+-+-+-+-+-+-+-+-+-+-+-+-+-+-+-+-+-+-+-+-+-+-+

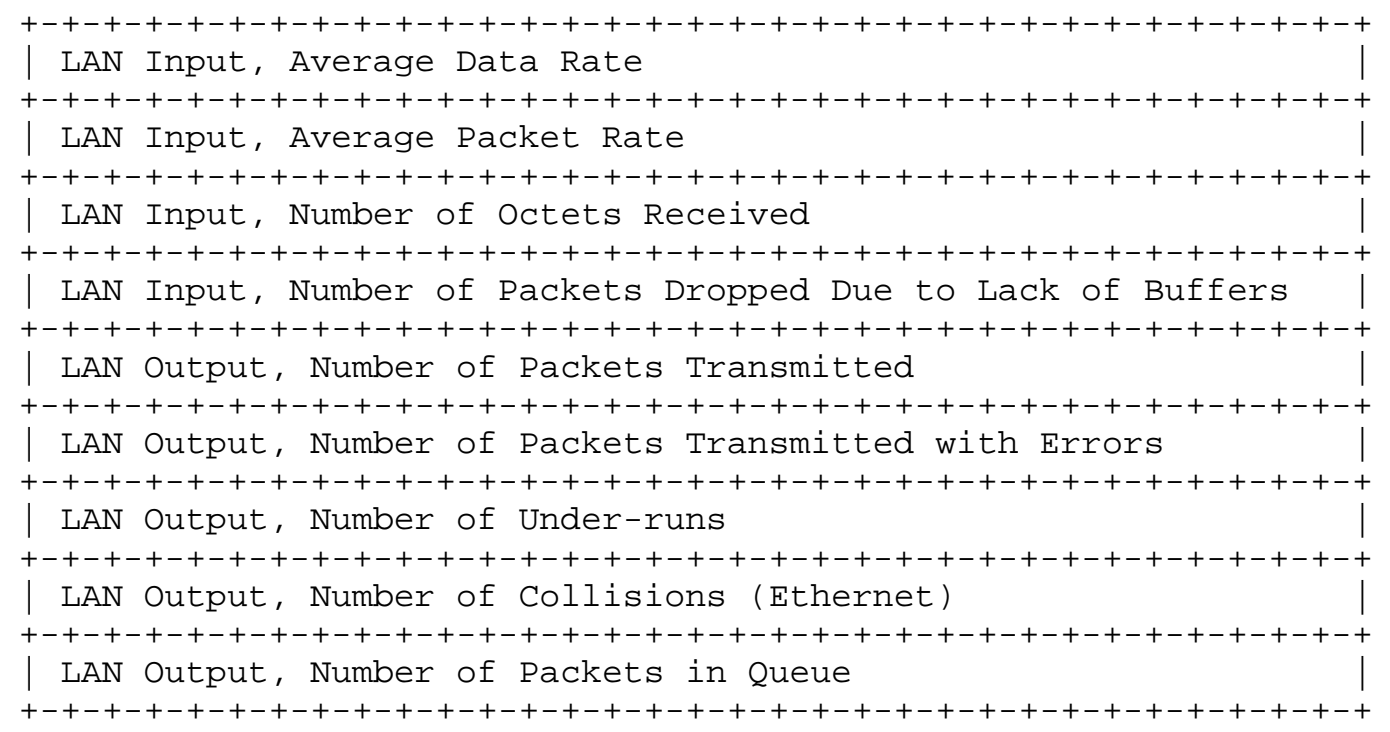


LAN Output, Queue Size
+-+-+-+-+-+-+-+-+-+-+-+-+-+-+-+-+-+-+-+-+-+-+-+-+-+-+-+-+-+-+-+-+
LAN Output, Number of Packets Dropped in Queue
+-+-+-+-+-+-+-+-+-+-+-+-+-+-+-+-+-+-+-+-+-+-+-+-+-+-+-+-+-+-+-+-+
LAN Output, Time of Last Packet Transmitted
+-+-+-+-+-+-+-+-+-+-+-+-+-+-+-+-+-+-+-+-+-+-+-+-+-+-+-+-+-+-+-+-+
LAN Output, Transmit Data Rate
+-+-+-+-+-+-+-+-+-+-+-+-+-+-+-+-+-+-+-+-+-+-+-+-+-+-+-+-+-+-+-+-+
LAN Output, Transmit Packet Rate
+-+-+-+-+-+-+-+-+-+-+-+-+-+-+-+-+-+-+-+-+-+-+-+-+-+-+-+-+-+-+-+-+
LAN Output, Number of Octets Transmitted
+-+-+-+-+-+-+-+-+-+-+-+-+-+-+-+-+-+-+-+-+-+-+-+-+-+-+-+-+-+-+-+-+
LAN, Number of Interface Resets
+-+-+-+-+-+-+-+-+-+-+-+-+-+-+-+-+-+-+-+-+-+-+-+-+-+-+-+-+-+-+-+-+

Note: Counts are cumulative since last counter reset. Time stamps are in milliseconds since last given event. Packet rates are in packets per second (averaged).

Summary Field Descriptions

For complete descriptions of the fields in the PPP Header, the LAN Extension Protocol Header, and the LAN Extension Remote Command Option, refer to the "Remote Command Options" section earlier in this document. The following table provides a summary of these fields for a LEX_RCMD_ACK packet that sends statistics to the host router.

Table 15 Field Values for LEX_RCMD_ACK Packet - Request Statistics

Field

Address/Control

Protocol-Type

Code

Identifier

Length

Option-Type

Option-Flags
Value

0xFF03 (Broadcast address/Unnumbered

information)

0x8041 (Control packet)

0x41 (LEX_RCMD_ACK packet)

The same value as that sent by the

LEX_RCMD_REQUEST packet

Minimum length $=120$ octets

Maximum length $=140$ octets

0x07 (Request statistics)

The LAN extension interface unit always sets this field to reflect the actions taken in response to the LEX_RCMD_ACK packet. Valid values:

* 0x01 - Reset serial statistics in the LAN extension interface unit

* 0x02 - Return serial statistics from the LAN extension interface unit

* 0x04 - Return LAN statistics from the LAN 
Option-Length

Option-Data extension interface unit

* 0x08 - Reset LAN statistics in the LAN extension interface unit

If both serial and LAN statistics are returned, the corresponding bits of this field should are set (that is, 0x06). The serial interface statistics appear in the response packet before the LAN statistics.

Minimum length $=116$ octets

Maximum length $=136$ octets

The Serial Interface Statistics Block, the LAN Interface Statistics Block, or both the Serial Interface and LAN Interface Statistics blocks. When the Option-Data contains both blocks, the Serial Interface Block precedes the LAN Interface Block. Statistics values that the LAN extension interface unit does not collect/support are retuned with a value of zero.

Implementation Notes

If the LAN extension interface unit does not implement the capability of sending the serial and LAN interface statistics blocks separately, the implementation may always return both statistics blocks (with the Option-Flags and Option-Length fields containing the appropriate corresponding values).

An implementation, such as a Token Ring LAN implementation, can collect a different set of statistics than shown above by defining a new statistics request type remote command option.

9.3 LEX RCMD_NAK/LEX RCMD_REJ - Request Statistics

The LAN extension interface unit sends a LEX_RCMD_NAK packet when the Statistics Request LEX_RCMD_REQUEST packet did not specify the type of statistics the host router wants. That is, the request's OptionFlags field equals zero.

Refer to the "Conditions for Sending PPP-LEX Packets" section earlier in this document for information on when the LAN extension interface unit sends a Request Statistics LEX_RCMD_REJ packet.

The frame format for the LEX_RCMD_NAK and LEX_RCMD_REJ packets is the same as that of the Statistics Request LEX_RCMD_REQUEST packet. Table 16 summarizes the appropriate field values for the Statistics Request LEX_RCMD_NAK and LEX_RCMD_REJ packets. 


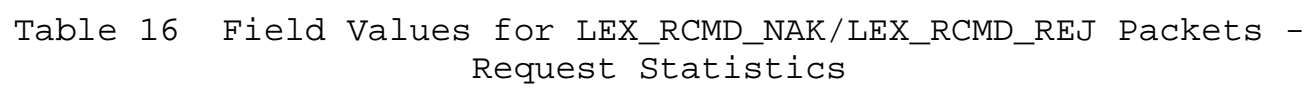

Field

Value

Address/Control

0xFF03 (Broadcast address/Unnumbered

information)

Protocol-Type

0x8041 (Control packet)

Code

Valid values:

* 0x42 (LEX_RCMD_NAK packet)

* 0x43 (LEX_RCMD_REJ packet)

Identifier

The same value as that sent by the

LEX_RCMD_REQUEST packet

Length

Option-Type

12 octets

0x07 (Request Statistics)

Option-Flags

The same value as that sent by the

Option-Length

LEX_RCMD_REQUEST packet

Option-Data

4 octets

None

10.0 Download Request

The host router sends a LEX_RCMD_REQUEST packet with an Option-Type of $0 \times 08$ to alert the LAN extension interface unit that the host router will be issuing a software download to Flash memory. When the LAN extension interface unit acknowledges the command, the host router starts sending download data. 


\subsection{LEX RCMD_REQUEST - Download Request}

Figure 14 shows a summary frame format of a LEX_RCMD_REQUEST packet for a Download Request remote command option. The host router transmits the fields from left to right.

Figure 14 LEX_RCMD_REQUEST Packet Frame Format - Download Request

PPP Header

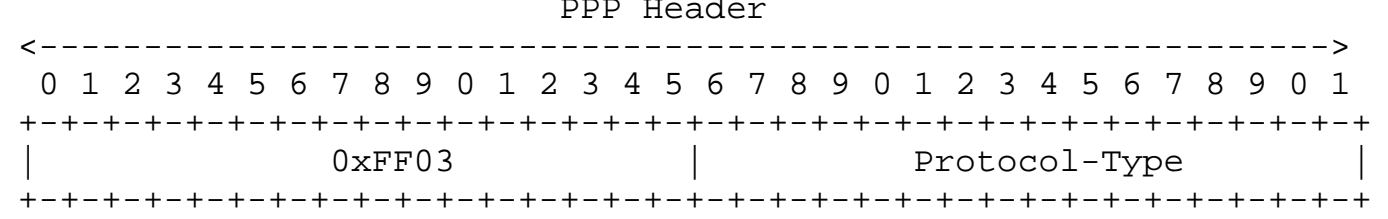

(2 octets)

(2)

LAN Extension Interface Protocol Header

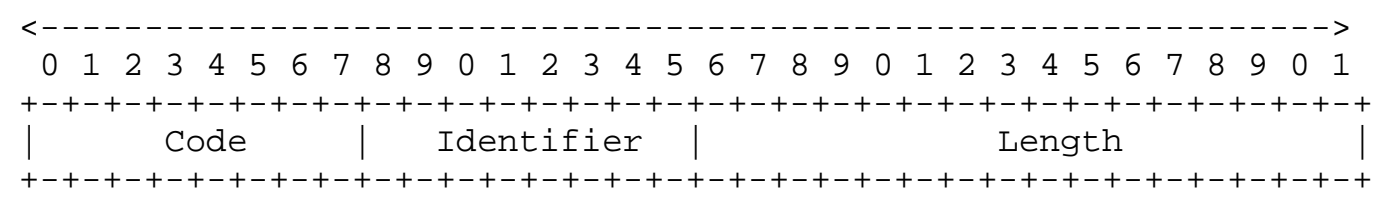

(1)

LAN Extension Interface Remote Command Option

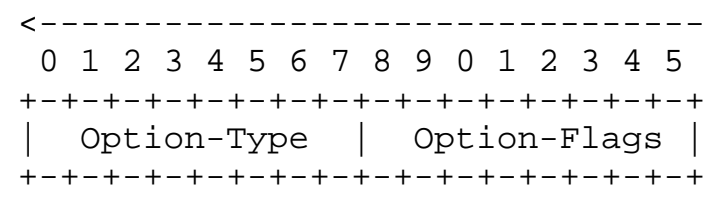

(1)

(1)

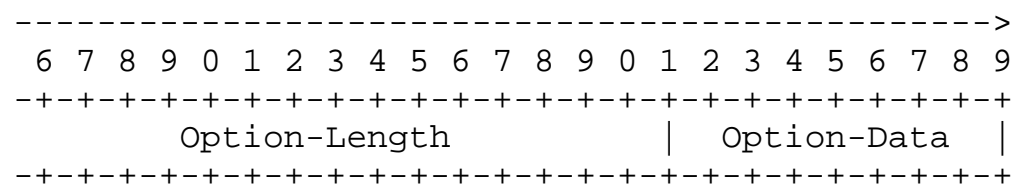

(2)

Where the Option-Data field contains the following fields:

$\begin{array}{llllllllllllllllllllllllllllllll}0 & 1 & 2 & 3 & 4 & 5 & 6 & 7 & 8 & 9 & 0 & 1 & 2 & 3 & 4 & 5 & 6 & 7 & 8 & 9 & 0 & 1 & 2 & 3 & 4 & 5 & 6 & 7 & 8 & 9 & 0 & 1\end{array}$ $+-+-+-+-+-+-+-+-+-+-+-+-+-+-+-+-+-+-+-+-+-+-+-+-+-+-+-+-+-+-+-+-+$ | Block Number (16 bits) | File Size (32 bits)... +-+-+-+-+-+-+-+-+-+-+-+-+-+-+-+-+-+-+-+-+-+-+-+-+-+-+-+-+-+-+-+-+ | Filename (Max. 64 octets)......

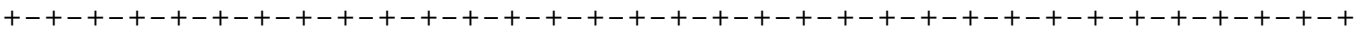




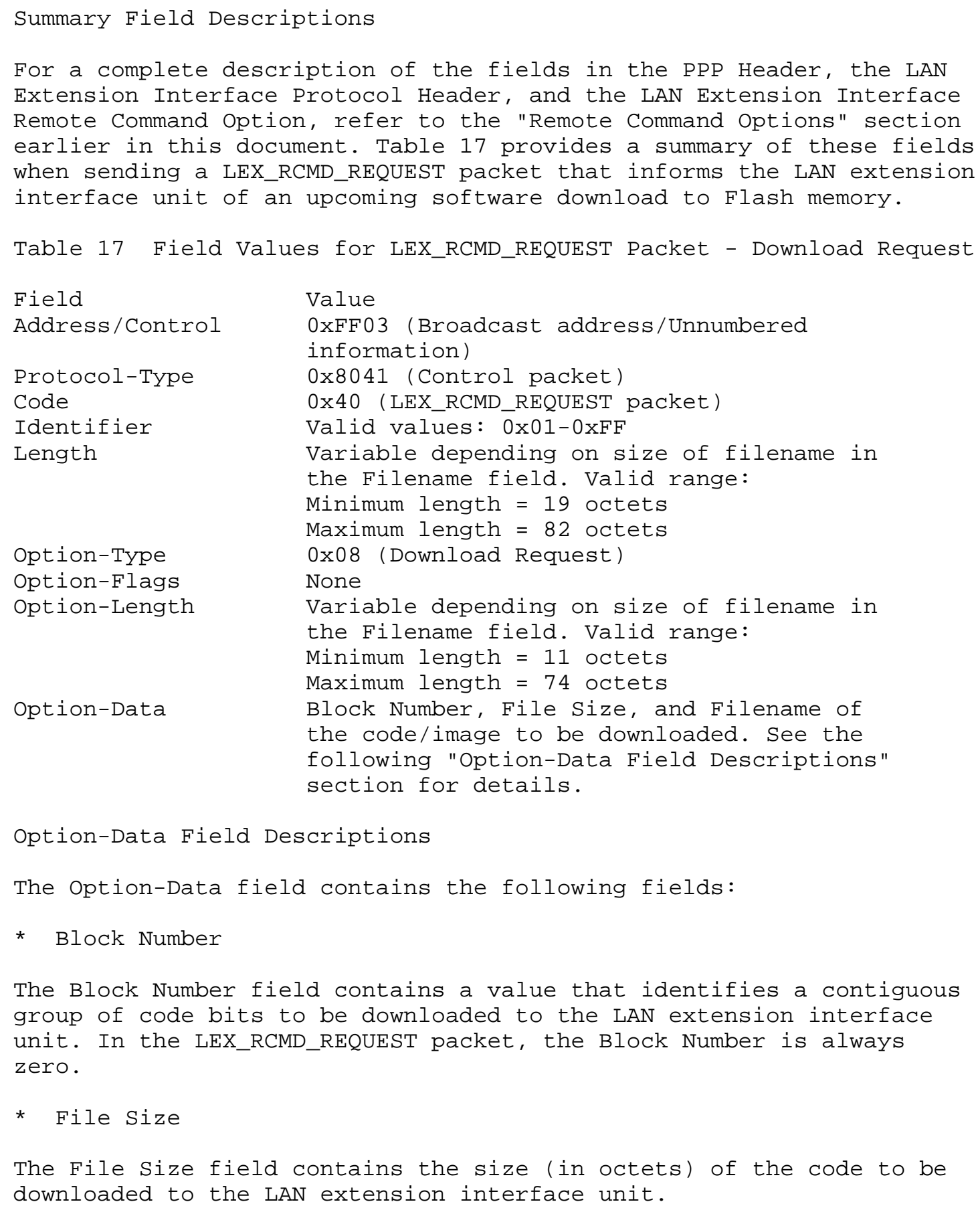


* Filename

The Filename field contains the name of the image to be transferred to the LAN extension interface unit.

10.2 Response Packets - Download Request

The following packets are valid responses to a Download Request LEX_RCMD_REQUEST packet:

* LEX_RCMD_ACK - Download Request

Refer to the "Conditions for Sending PPP-LEX Packets" section earlier in this document for more information on when the LAN extension interface unit sends this packet.

* LEX_RCMD_NAK - Download Request

The LAN extension interface unit sends a Download Request LEX_RCMD_NAK packet when the LAN Extension interface unit is currently programming Flash, when the File size field value in the request is greater than the unit's available Flash bytes, when the LAN extension interface unit is out of memory, or when the Block Number field value in the request is not zero.

* LEX_RCMD_REJ - Download Request

Refer to the "Conditions for Sending PPP-LEX Packets" section earlier in this document for more information on when the LAN extension interface unit sends this packet.

Table 18 summarizes the field values of the Download Request LEX_RCMD_ACK, LEX_RCMD_NAK, and LEX_RCMD_REJ packets.

Table 18 Field Values for Response Packets - Download Request

Field

Address/Control

Protocol-Type

Code

Identifier

Length
Value

OxFF03 (Broadcast address/Unnumbered

information)

0x8041 (Control packet)

Valid values:

* 0x41 (LEX_RCMD_ACK packet)

* 0x42 (LEX_RCMD_NAK packet)

* 0x43 (LEX_RCMD_REJ packet)

The same value as that sent by the LEX_RCMD_REQUEST packet

The same value as that sent by the LEX_RCMD_REQUEST packet 


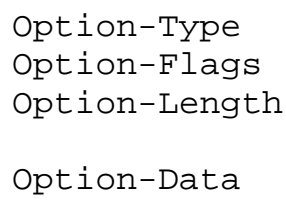

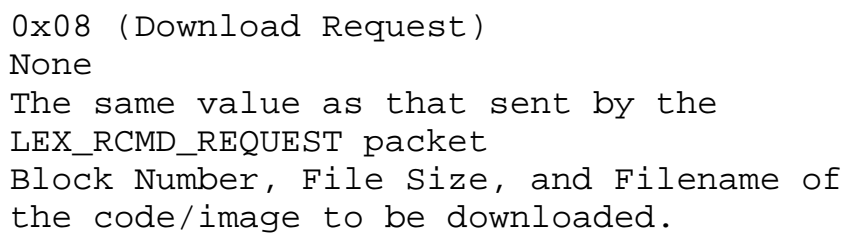

\subsection{Download Data}

Once the host router receives a Download Request LEX_RCMD_ACK packet from the LAN extension interface unit, the host router downloads the data to be written to Flash memory by sending a LEX_RCMD_REQUEST packet with an Option-Type of $0 \times 09$. The host router sends multiple Download Data LEX_RCMD_REQUEST packets, each with 512 octets of Option-Data containing the binary data to be programmed into Flash memory.

A packet containing Option-Data with less than 512 octets signals the end of the download data (that is, the end of tile). If the size of the file being downloaded is an even multiple of 512 bytes, then the last packet transferred should have an Option-Length of 6 . That is, the last packet transferred should have Option-Data containing the Block Number field value but no data. A Block Number field value without data alerts the LAN extension interface unit that this is the last block to be transmitted.

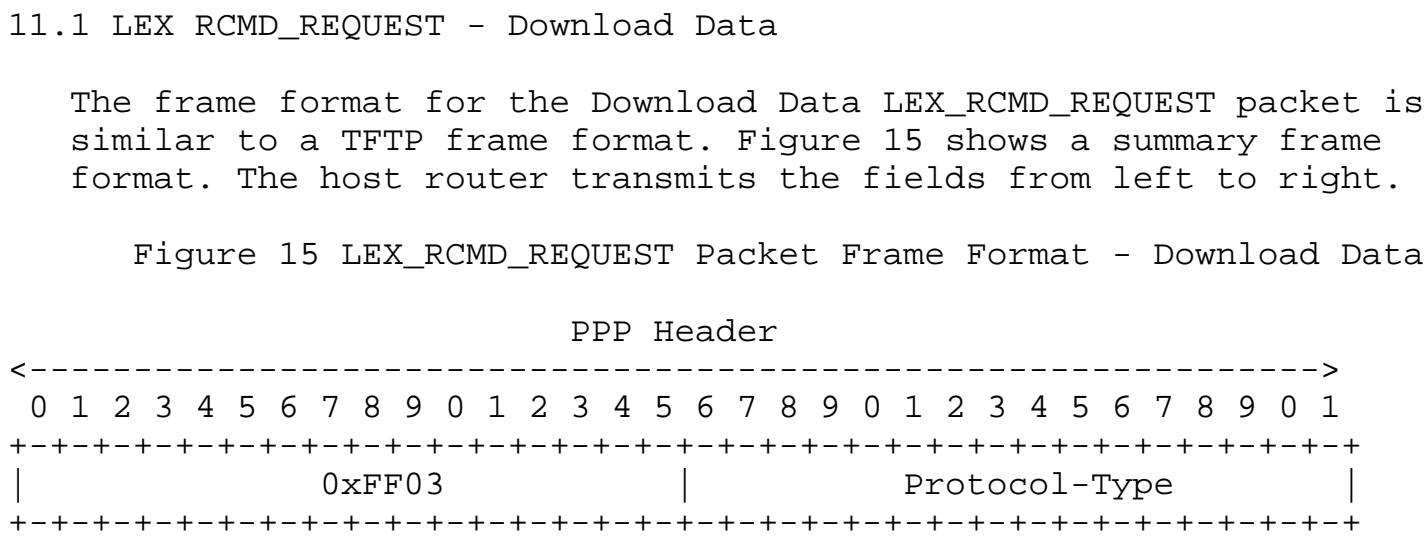


LAN Extension Interface Protocol Header

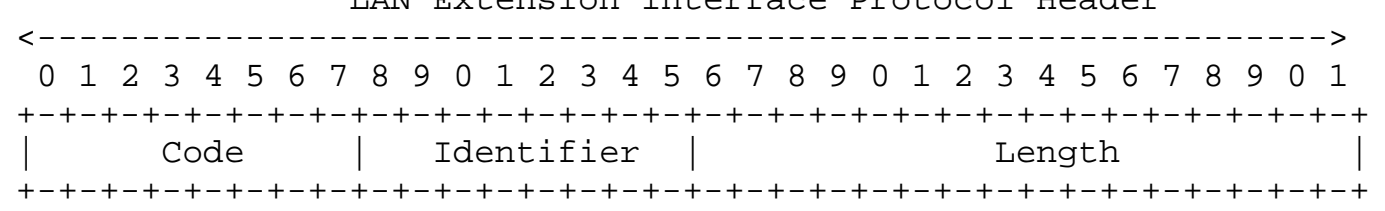

(1)

LAN Extension Interface Remote Command Option

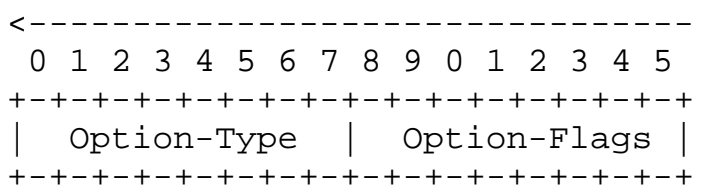

(1)

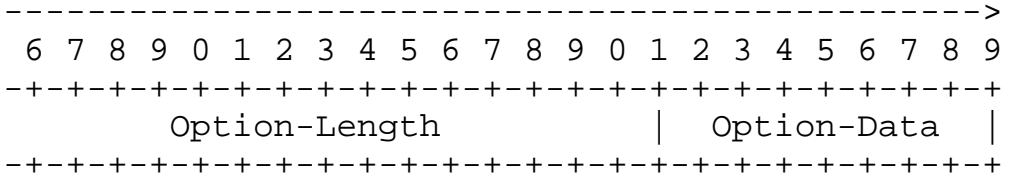

(2)

Where the Option-Data field contains the following fields:

$\begin{array}{llllllllllllllllllllllllllllllll}0 & 1 & 2 & 3 & 4 & 5 & 6 & 7 & 8 & 9 & 0 & 1 & 2 & 3 & 4 & 5 & 6 & 7 & 8 & 9 & 0 & 1 & 2 & 3 & 4 & 5 & 6 & 7 & 8 & 9 & 0 & 1\end{array}$ +-+-+-+-+-+-+-+-+-+-+-+-+-+-+-+-+-+-+-+-+-+-+-+-+-+-+-+-+-+-+-+-+ Block Number (16 bits) |
+-+-+-+-+-+-+-+-+-+-+-+-+-+-+-+-+
Binary Download Data (Max. 512 octets) ....

Summary Field Descriptions

For a complete descriptions of the fields in the PPP Header, the LAN Extension Interface Protocol Header, and the LAN Extension Interface Remote Command Option, refer to the "Remote Command Options" section earlier in this document. The following table provides a summary of these fields when sending a LEX_RCMD_REQUEST packet that download software to the LAN extension interface unit.

Table 19 Field Values for LEX_RCMD_REQUEST Packet - Download Data

Field

Address/Control

Protocol-Type

Code
Value

0xFF03 (Broadcast address/Unnumbered information)

0x8041 (Control packet)

0x40 (LEX_RCMD_REQUEST packet) 


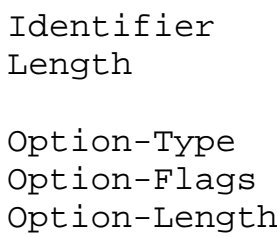

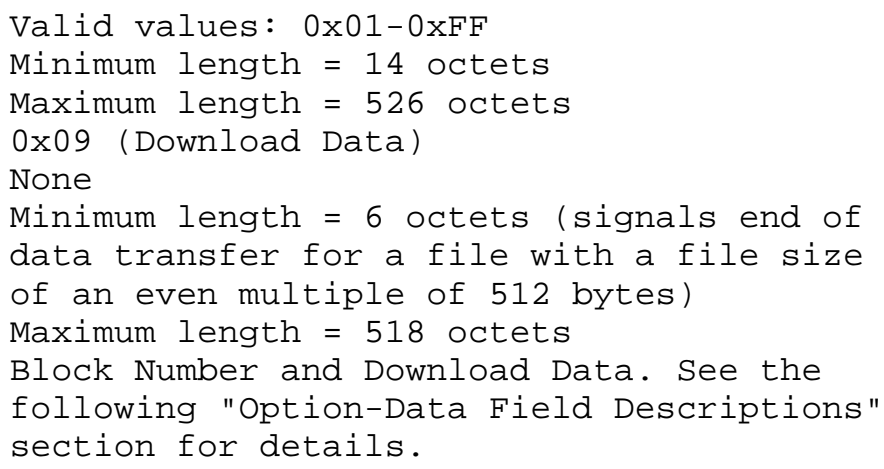

Option-Data Field Descriptions

The following Option-Data fields provide the LAN extension interface unit with download data:

* Block Number

The Block Number identifies the contiguous group of code bits to be downloaded. The host router automatically generates this number. The first Download Data packet gets a block number of one. The host router increments this Block Number value by one with every Download Data LEX_RCMD_REQUEST packet sent to the LAN extension interface unit. The maximum value of the Block Number is dependent on the unit's Flash memory size.

* Download Data

The Download Data are the actual code bits being downloaded to the LAN extension interface unit. A maximum of 512 octets of download data can sent in one Download Data LEX_RCMD_REQUEST packet.

11.2 Response Packets - Download Data

The following packets are valid responses to a Download Data LEX_RCMD_REQUEST packet:

* LEX_RCMD_ACK - Download Data

Refer to the "Conditions for Sending PPP-LEX Packets" section earlier in the document for more information on this packet. 
* LEX_RCMD_NAK - Download Data

The LAN extension interface unit sends a Download Data LEX_RCMD_NAK packet when the LAN extension interface unit is not in the proper state or when accepting the data will over-run the download buffer.

* LEX_RCMD_REJ - Download Data

Refer to the "Conditions for Sending PPP-LEX Packets" section earlier in the document for more information on this packet.

Table 20 summarizes the field values of the Download Data LEX_RCMD_ACK, LEX_RCMD_NAK, and LEX_RCMD_REJ packets.

Table 20 Field Values for Response Packets - Download Data

$\begin{array}{ll}\text { Field } & \text { Value } \\ \text { Address/Control } & \text { OxFF03 (Broadcast address/Unnumbered } \\ & \text { information) } \\ \text { Protocol-Type } & \text { 0x8041 (Control packet) } \\ \text { Code } & \text { Valid values: } \\ & \star 0 \times 41 \text { (LEX_RCMD_ACK packet) } \\ & \star 0 \times 42 \text { (LEX_RCMD_NAK packet) } \\ & \star 0 \times 43 \text { (LEX_RCMD_REJ packet) } \\ \text { Identifier } & \text { The same value as that sent by the } \\ & \text { LEX_RCMD_REQUEST packet } \\ \text { Length } & \text { 14 octets } \\ \text { Option-Type } & \text { Ox09 (Download Data) } \\ \text { Option-Flags } & \text { None } \\ \text { Option-Length } & 6 \text { octets } \\ \text { Option-Data } & \text { Block Number only }\end{array}$

12.0 Download Status

Upon completion of a Download Data sequence, the host router issues a LEX_RCMD_REQUEST packet with an Option-Type of 0x0A to request status of the success or failure of the download. A bad checksum of the image or a malfunctioning Flash memory could cause the download to fail. 


\subsection{LEX RCMD_REQUEST - Download Status}

Figure 16 shows the frame format summary of a LEX_RCMD_REQUEST packet for a Download status remote command option. The host router transmits the fields from left to right.

Figure 16 LEX_RCMD_REQUEST Packet Frame Format - Download Status

PPP Header

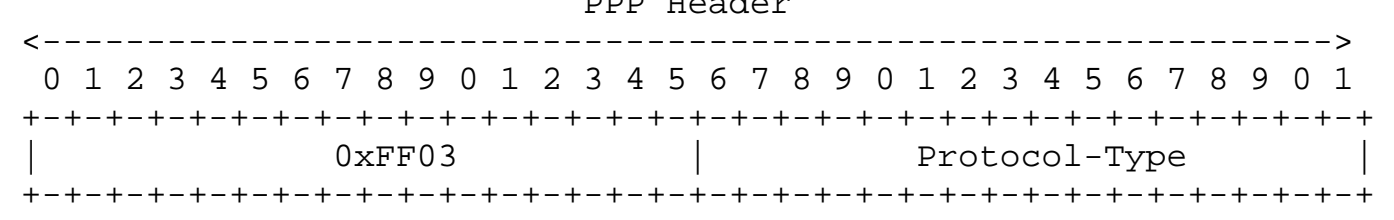

(2 octets)

(2)

LAN Extension Interface Protocol Header

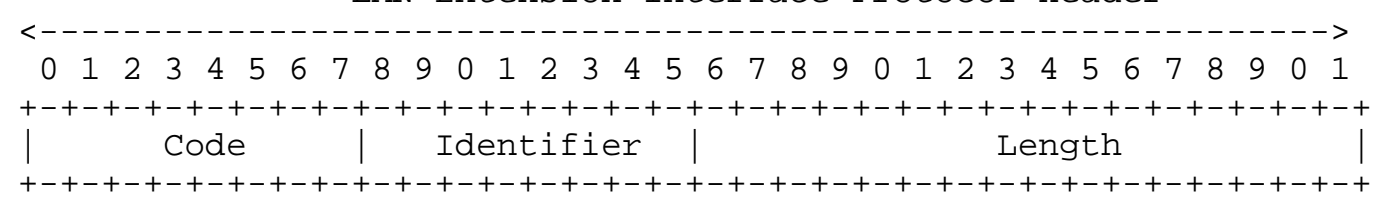

(1)

LAN Extension Interface Remote Command Option

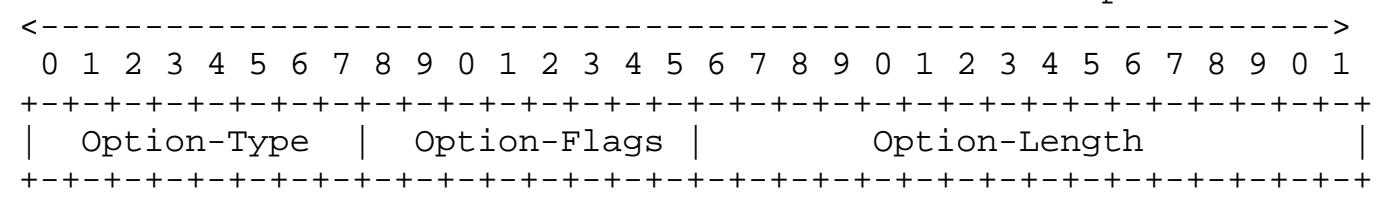

(2)

Summary Field Descriptions

For a complete descriptions of the fields in the PPP Header, the LAN Extension Interface Protocol Header, and the LAN Extension Interface Remote Command Option, refer to the "Remote Command Options" section earlier in this document. Table 21 provides a summary of these fields when sending a LEX_RCMD_REQUEST packet that requests the download status of the LAN extension interface unit.

Table 21 Field Values for LEX_RCMD_REQUEST Packet - Download Status

Field Value

Address/Control 0xFF03 (Broadcast address/Unnumbered

Protocol-Type 0x8041 (Control packet)

Code 0x40 (LEX_RCMD_REQUEST packet) 


$\begin{array}{ll}\text { Identifier } & \text { Valid values: 0x01-0xFF } \\ \text { Length } & 12 \text { octets } \\ \text { Option-Type } & \text { 0x0A (Download Status) } \\ \text { Option-Flags } & \text { None } \\ \text { Option-Length } & 4 \text { octets } \\ \text { Option-Data } & \text { None } \\ .2 \text { LEX RCMD_REQUEST - Download Status }\end{array}$

When the LAN extension interface unit correctly receives the Download Status LEX_RCMD_REQUEST packet, it returns a LEX_RCMD_ACK packet containing the appropriate status information in the Option-Data field.

Figure 17 shows the frame format for the Download Status LEX_RCMD_ACK packet. The LAN extension interface unit transmits the fields from left to right.

Figure 17 LEX_RCMD_ACK Packet Frame Format - Download Status PPP Header

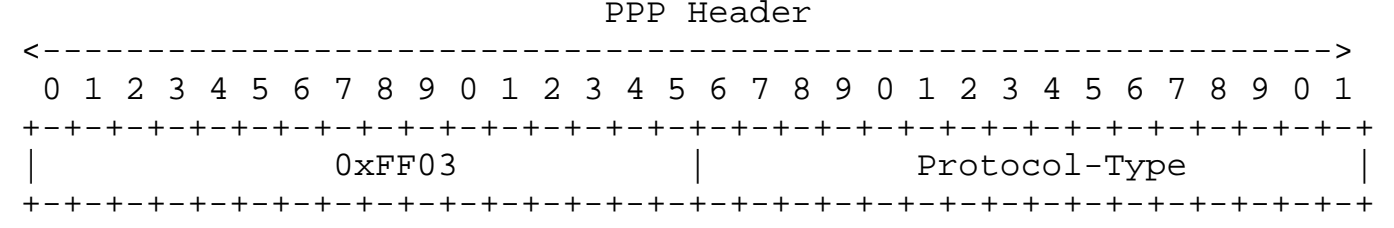

(2 octets)

(2)

LAN Extension Interface Protocol Header

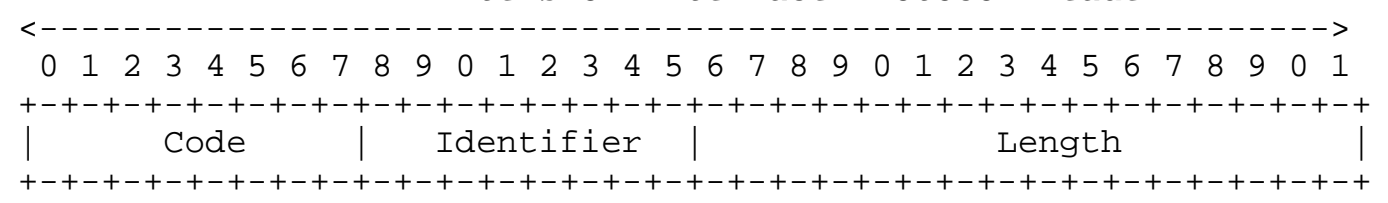

(1)

(1)

(2) 
LAN Extension Interface Remote Command Option

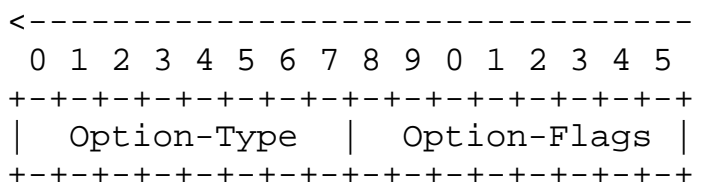

(1)

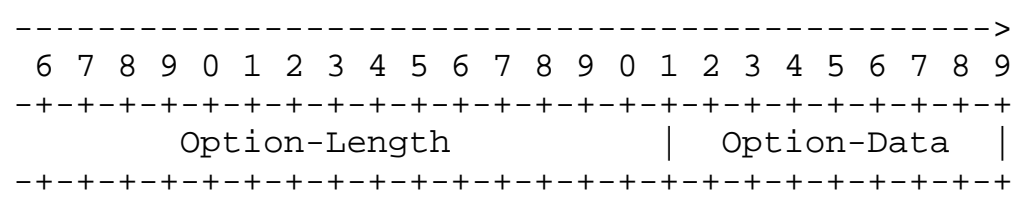

(2)

Where the Option-Data field contains the following fields:

$\begin{array}{llllllllllllllll}0 & 1 & 2 & 3 & 4 & 5 & 6 & 7 & 8 & 9 & 0 & 1 & 2 & 3 & 4 & 5\end{array}$

+-+-+-+-+-+-+-+-+-+-+-+-+-+-+-+-+
Status $(16$ bits $)$

Summary Field Descriptions

For a complete descriptions of the fields in the PPP Header, the LAN Extension Interface Protocol Header, and the LAN Extension Interface Remote Command Option, refer to the "Remote Command Options" section earlier in this document. Table 22 provides a summary of these fields when sending a status information to the host router.

Table 22 Field Values for LEX_RCMD_ACK Packet - Download Status

Field
Address/Control
Protocol-Type
Code
Identifier
Length
Option-Type
Option-Flags
Option-Length
Option-Data

Value

0xFF03 (Broadcast address/Unnumbered

information)

0x8041 (Control packet)

0x41 (LEX_RCMD_ACK packet)

The same value as that sent by the

LEX_RCMD_REQUEST packet

14 octets

0x0A (Download Status)

None

6 octets

Status information. See the following

"Option-Data Field Descriptions" section

for details. 


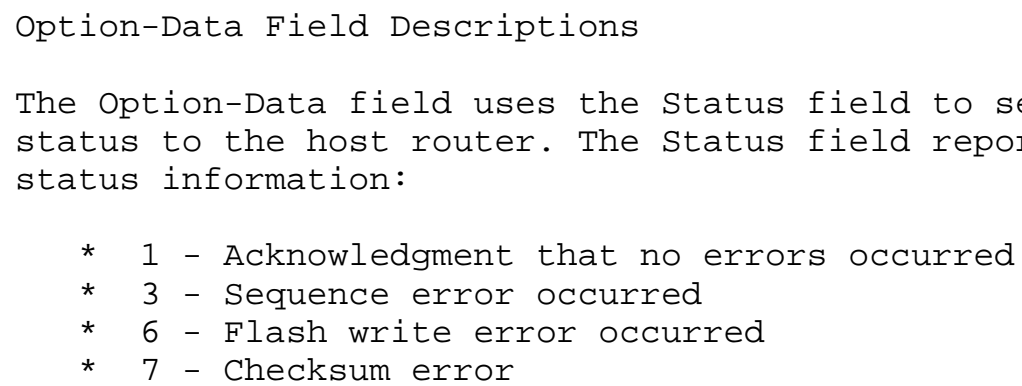

12.3 LEX RCMD_NAK/LEX RCMD_REJ - Download Status

Refer to the "Conditions for Sending PPP-LEX Packets" section earlier in this document for information on when the LAN extension interface unit sends a Download Status LEX_RCMD_NAK packet and Download Status LEX_RCMD_REJ packet.

The frame format for these two response packets mirror the frame format of the Download Status LEX_RCMD_REQUEST packet. Table 23 summarizes the field values of the Download Status LEX_RCMD_NAK and LEX_RCMD_REJ packets.

Table 23 Field Values for LEX_RCMD_NAK/LEX_RCMD_REJ Packets - Download Status

$\begin{array}{ll}\text { Field } & \text { Value } \\ \text { Address/Control } & \text { OxFF03 (Broadcast address/Unnumbered } \\ & \text { information) } \\ \text { Protocol-Type } & \text { 0x8041 (Control packet) } \\ \text { Code } & \text { Valid values: } \\ & \star 0 \times 42 \text { (LEX_RCMD_NAK packet) } \\ & \star 0 \times 43 \text { (LEX_RCMD_REJ packet) } \\ \text { Identifier } & \text { The same value as that sent by the } \\ & \text { LEX_RCMD_REQUEST packet } \\ \text { Length } & \text { 12 octets } \\ \text { Option-Type } & \text { 0x0A (Download Status) } \\ \text { Option-Flags } & \text { None } \\ \text { Option-Length } & 4 \text { octets } \\ \text { Option-Data } & \text { None }\end{array}$

\subsection{Inventory Request}

The host router sends a LEX_RCMD_REQUEST packet with an Option-Type of $0 x 0 B$ to the LAN extension interface unit to request inventory information. The host router may use this remote command option to accomplish the following: 
* Distinguish between different versions of the LAN extension interface unit for determining their capabilities

* Determine whether a new version of Flash code should be downloaded

13.1 LEX RCMD_REQUEST - Inventory Request

Figure 18 shows the frame format for a LEX_RCMD_REQUEST packet with an Inventory Request remote command option. The host router transmits the fields from left to right.

Figure 18 LEX_RCMD_REQUEST Packet Frame Format - Inventory Request

PPP Header

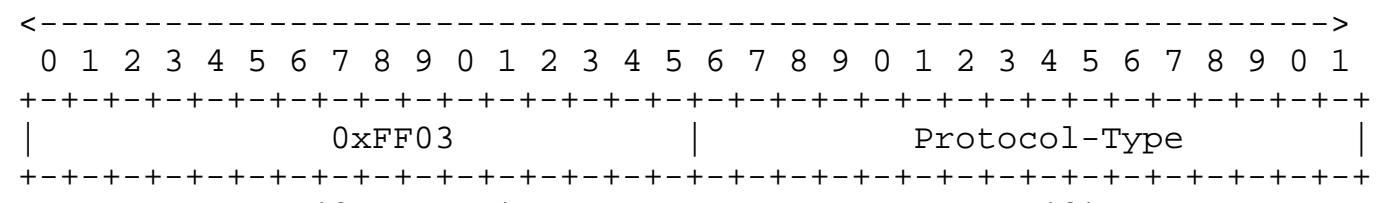

(2 octets)

LAN Extension Interface Protocol Header

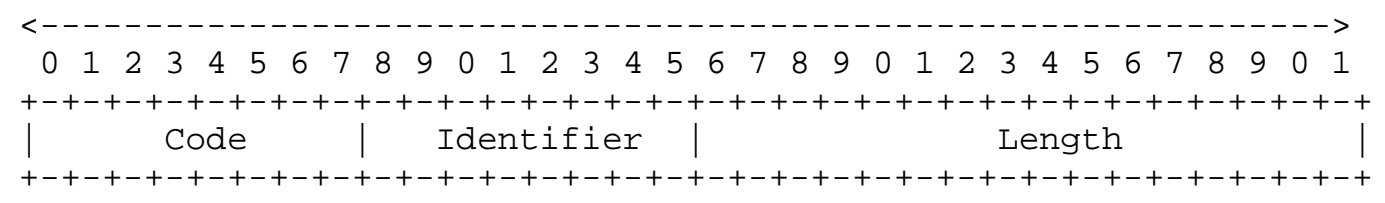

(1)

LAN Extension Interface Remote Command Option

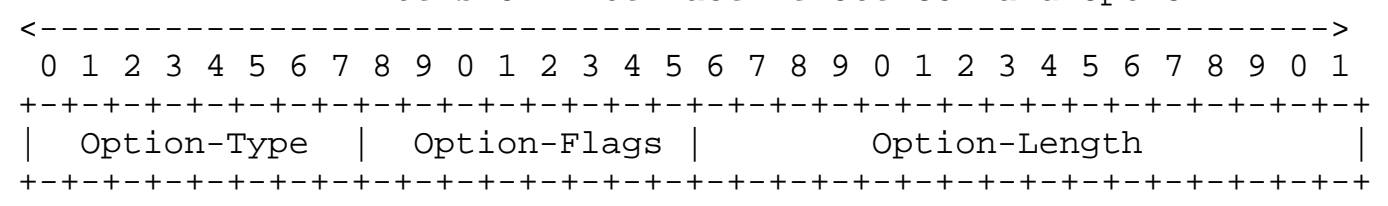

(1)

(2)

Summary Field Descriptions

For a complete description of the fields in the PPP Header, the LAN Extension Interface Protocol Header, and the LAN Extension Interface Remote Command Option, refer to the "Remote Command Options" section earlier in this document. Table 24 provides a summary of these fields when sending a LEX_RCMD_REQUEST packet that requests inventory information. 
Table 24 Field Values for LEX_RCMD_REQUEST Packet-Inventory Request

$\begin{array}{ll}\text { Field } & \text { Value } \\ \text { Address/Control } & \text { 0xFF3 (Broadcast address/Unnumbered } \\ & \text { information) } \\ \text { Protocol-Type } & \text { 0x8041 (Control packet) } \\ \text { Code } & \text { 0x40 (LEX_RCMD_REQUEST packet) } \\ \text { Identifier } & \text { Valid values: 0x01-0xFF } \\ \text { Length } & 12 \text { octets } \\ \text { Option-Type } & \text { 0x0B (Inventory Request) } \\ \text { Option-Flags } & \text { None } \\ \text { Option-Length } & 4 \text { octets } \\ \text { Option-Data } & \text { None }\end{array}$

13.2 LEX RCMD_ACK - Inventory Request

When it correctly receives the Inventory Request remote command option and retrieves the inventory information, the LAN extension interface unit responds with an acknowledgment. This acknowledgment contains the requested inventory information in the option-Data field.

Figure 19 shows the frame format for the Inventory Request LEX_RCMD_ACK packet.

Figure 19 LEX_RCMD_ACK Packet Frame Format - Inventory Request

PPP Header

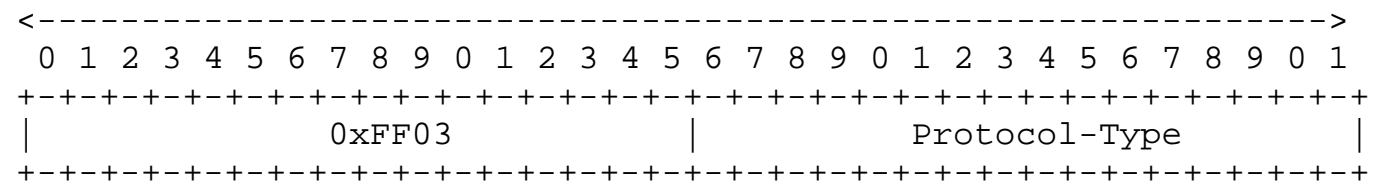

(2 octets)

LAN Extension Interface Protocol Header

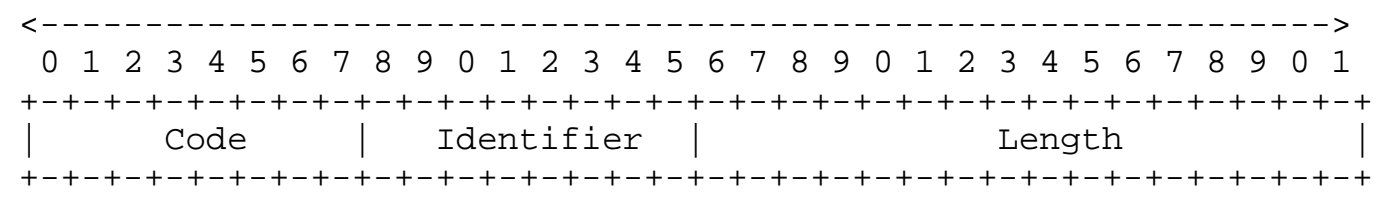

(1)

(2) 
LAN Extension Interface Remote Command Option

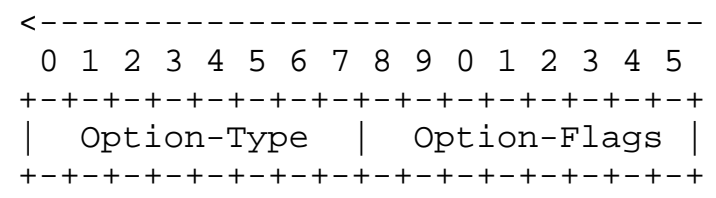

(1)

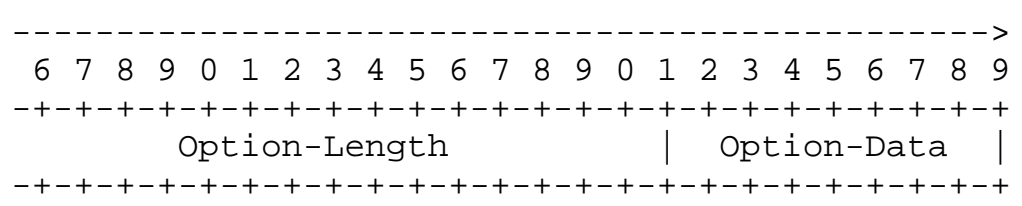

(2)

Where the Option-Data field contains the following fields:

(32 bits)

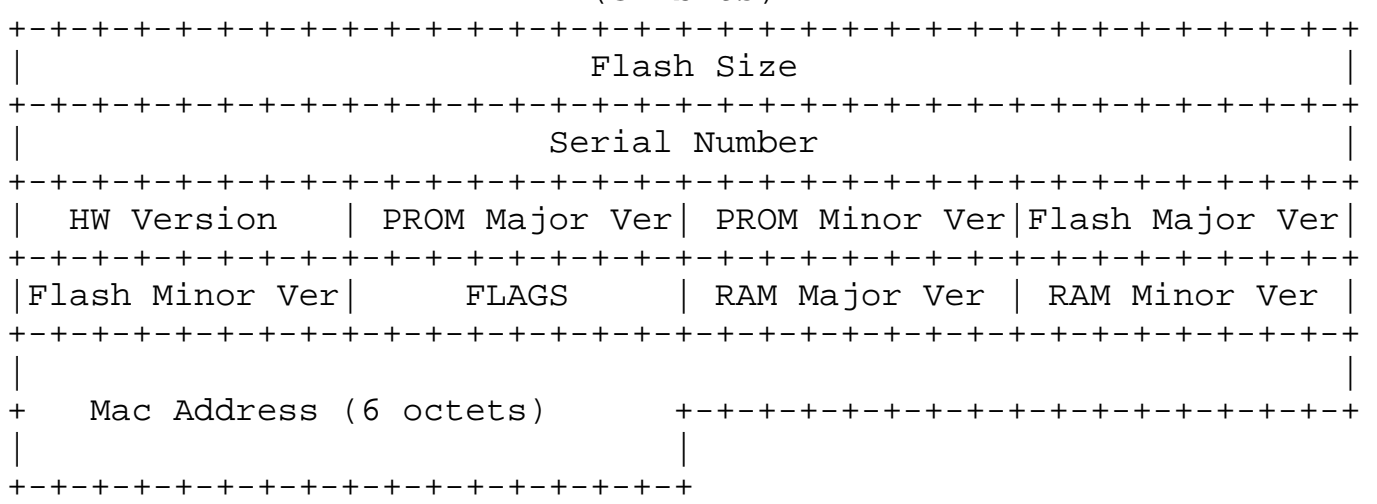

Summary Field Descriptions

For a complete description of the fields in the PPP Header, the LAN Extension Interface Protocol Header, and the LAN Extension Interface Remote Command Option, refer to the "Remote Command Options" section earlier in this document. Table 25 provides a summary of these fields when acknowledging a LEX_RCMD_REQUEST packet that requests inventory information.

Table 25 Field Values for LEX_RCMD_ACK Packet - Inventory Request

Field Value

Address/Control 0xFF03 (Broadcast address/Unnumbered

Protocol-Type 0x8041 (Control packet)

Code 0x41 (LEX_RCMD_ACK packet) 


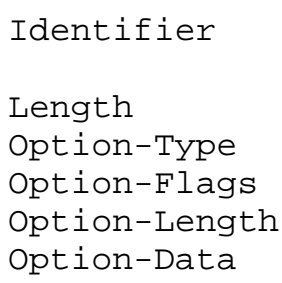

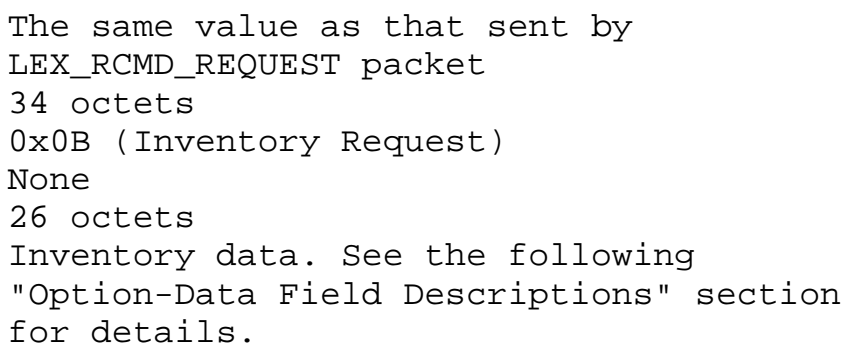

Option-Data Field Descriptions

The Option-Data field of the LEX_RCMD_ACK packet uses the following fields to send inventory data to the host router about the LAN extension interface unit.

* Flash Size

This field contains the LAN extension interface unit's Flash size in bytes.

* Serial Number

This field provides the LAN extension interface unit's serial number. This is an unassigned, 32-bit number.

* HW Version

This field contains the version number of the LAN extension interface hardware unit.

* PROM Major Ver

This field contains part of the version number of the PROM image. "Major" indicates which major software release this revision belongs to. For example, if the PROM version number is 1.2 , then the major version number is 1 .

* PROM Minor Ver

This field contains part of the version number of the PROM image. "Minor" indicates which minor software release this revision belongs to. For example, if the PROM version number is 1.2 , then the minor version number is 2 . 
* Flash Major Ver

This field contains the "major" version number of the Flash image. For example, if the Flash image version number is 1.2 , then the major version number is 1 .

* Flash Minor Ver

This field contains the "minor" version number of the Flash image. For example, if the Flash image version number is 1.2 , then the minor version number is 2 .

* FLAGS

FLAGS report the boot status of the LAN extension interface unit. The flags are as follows:

- 0x01 - Running PROM image

- 0x02 - Running Flash image

- 0x04 - PROM image passed checksum

- 0x08 - Flash image passed checksum

* RAM Major Ver

This field contains the "major" version number of the running image in RAM. For example, if the RAM image version number is 1.2 , then the major version number is 1 .

* RAM Minor Ver

This field contains the "minor" version number of the running image in RAM. For example, if the RAM image version number is 1.2 , then the minor version number is 2 .

* MAC Address

The MAC Address is the LAN extension interface unit's burned-in MAC address in canonical format. This field is six octets.

13.3 LEX RCMD_NAK/LEX RCMD_REJ - Inventory Request

Refer to the "Conditions for Sending PPP-LEX Packets" section earlier in this document for information on when the LAN extension interface unit sends an Inventory Request LEX_RCMD_NAK packet and Inventory Request LEX_RCMD_REJ packet.

The frame format of these two response packets mirrors that of the request. Table 26 summarizes the field values for such Inventory 


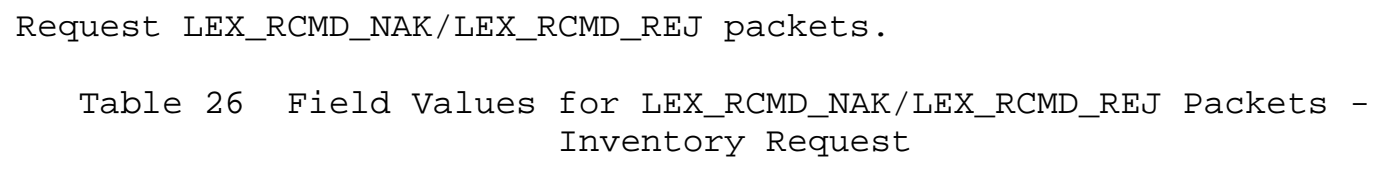

Field

Address/Control

Protocol-Type

Code

Identifier

Length

Option-Type

Option-Flags

Option-Length

Option-Data

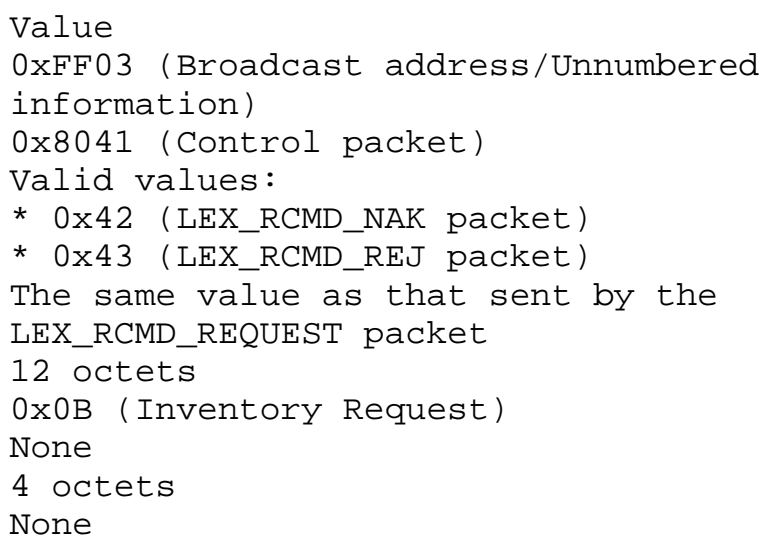

14.0 LAN Extension Interface Protocol Data Packets

When the PPP-LEX NCP is in an "opened" state, the LAN extension interface unit and the host router also exchange PPP-LEX data packets (as well as control packets). There is only one type of PPP-LEX data packet. This data packet is a subset of the PPP-BCP packet format.

The format subsetting is such that a PPP-BCP implementation will successfully process a LAN extension interface protocol packet. The differences are as follows:

* LAN ID field will not be present.

* LAN FCS field will never be present (that is, the F flag will always be off [=0]).

* LAN ID flag (I) will always be off.

* Pad field for the serial link will never be present, and the count field will be 0 .

For detailed information on PPP-BCP packets, refer to the "PPP Bridging Control Protocol (BCP)" RFC. (References, [2])

\subsection{Frame Format}

Figure 20 shows the frame format for a PPP-LEX data packet. The MAC frame is transferred except for the FCS field. The LAN extension interface unit computes the FCS for packets transferred to the LAN and strips the FCS for packets destined for the host router. 
Figure 20 PPP-LEX Data Packet Frame Format

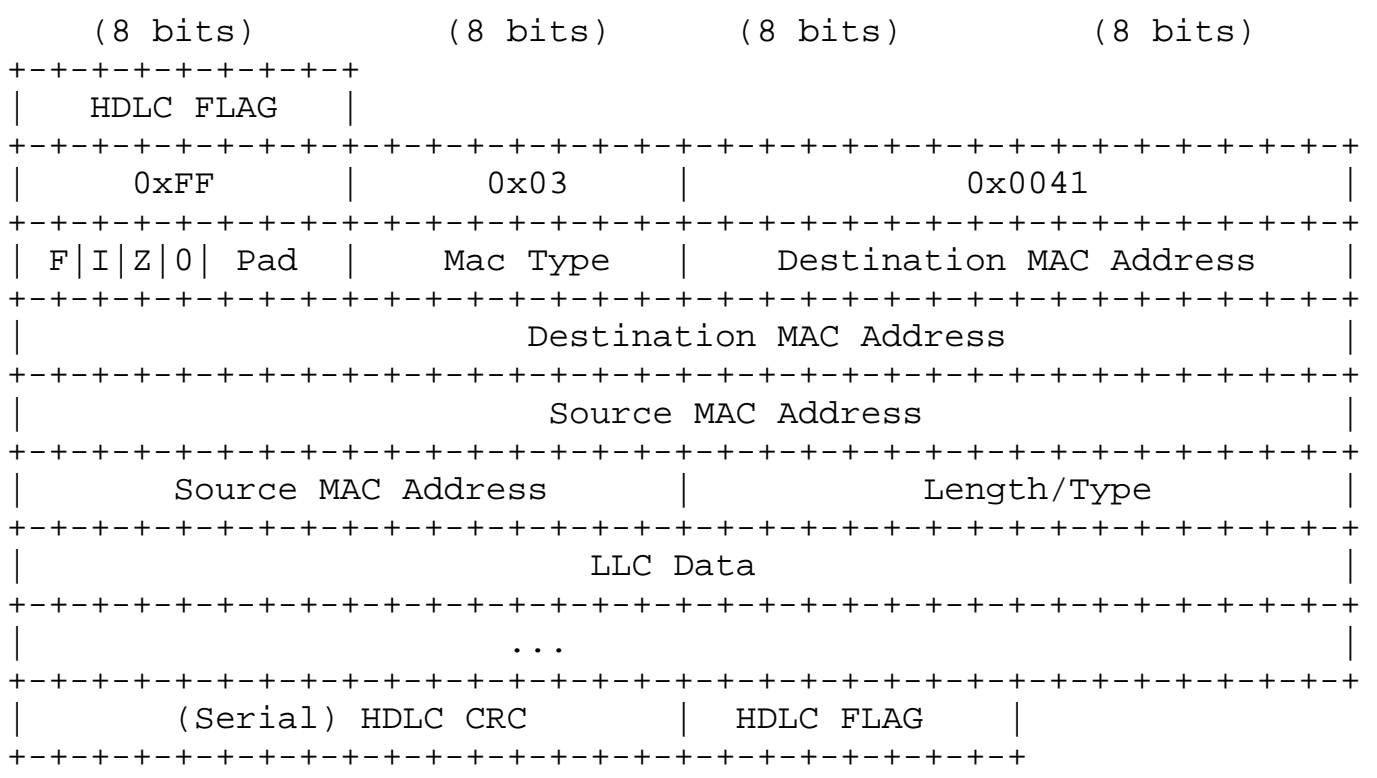

14.2 Summary Field Descriptions

HDLC FLAG

HDLC Frame delimiter.

OXFF

This Address field contains the broadcast address.

$0 \times 03$

This Control field contains unnumbered information.

$0 \times 0041$

This field contains the IETF-assigned protocol type value for a PPP-LEX data packet. In this case this field will always contain $0 \times 0041$.

Flags

The flags $F, I, Z, O$ have the following meanings: 
* $F$ : Set bit $F$ if the LAN FCS field is present. Because PPP-LEX data packets do not contain the LAN FCS field, this bit should not be set (field=0).

* I: Set bit I if the LAN ID field is present. Because PPP-LEX data packets do not contain the field, this bit should not be set $(f i e l d=0)$.

* Z: Set bit Z if IEEE 802.3 Pad must be zero filled to minimum size.

* 0: Reserved, must be zero.

Pad

Any PPP frame may have padding inserted in the Optional Data Link Layer Padding field. The value tells the receiving system how many pad octets to strip off. The LAN extension interface protocol does not support the Optional Data Link Layer Padding field, so the value of this field should be zero.

MAC Type

This field contains the most up-to-date value of the MAC type as specified in the most recent "Assigned Numbers" RFC. The current value is as follows:

* 1: IEEE 802.3/Ethernet with canonical addresses

Destination MAC Address

This field is 6 octets and contains the MAC address of the destination system as defined by IEEE. The MAC Type field defines the bit ordering.

Source MAC Address

This field is 6 octets and contains the MAC address of the destination system as defined by IEEE. The MAC Type field defines the bit ordering.

Length/Type

This field is any Ethernet protocol type (See RFC 1700 in the references "Assigned Numbers"). For IEEE 802.3 frames, this is a length field. 


\section{LLC Data}

This field is the remainder of the MAC frame which is (or would be if it were present) protected by the LAN FCS.

(Serial) HDLC CRC

This is a 16 bit Cyclic Redundancy Check field.

For complete information on the above fields and their relationship to PPP-BCP packets, refer to the "PPP Bridging Control Protocol $(\mathrm{BCP})$ " RFC. (References, [2])

Notes

1. The LAN extension interface protocol does allow the segmentation of individual LAN packets across the serial link. Each LAN packet must be transmitted across the serial link as one PPP-LEX encapsulation.

2. MAC addresses in PPP-LEX packets should be in canonical format.

References

[1] Simpson, W., "The Point-To-Point Protocol (PPP) for the Transmission of Multi-protocol Datagrams over Point-To-Point Links", RFC 1331, Daydreamer, May 1992.

[2] Baker, F., and R. Bowen, "PPP Bridging Control Protocol (BCP)", RFC 1638, ACC, IBM, June 1994.

[3] Lloyd, B., and W. Simpson, "PPP Authentication Protocols", RFC 1334, Lloyd \& Associates, Daydreamer, October 1992.

[4] Reynolds, J., and J. Postel, "Assigned Numbers", STD 2, RFC 1700, USC/Information Sciences Institute, October 1994.

[5] Reynolds, J., and J. Postel, "Standard for the transmission of IP datagrams over IEEE 802 networks", RFC 1042, USC/Information Sciences Institute, February 1988. 


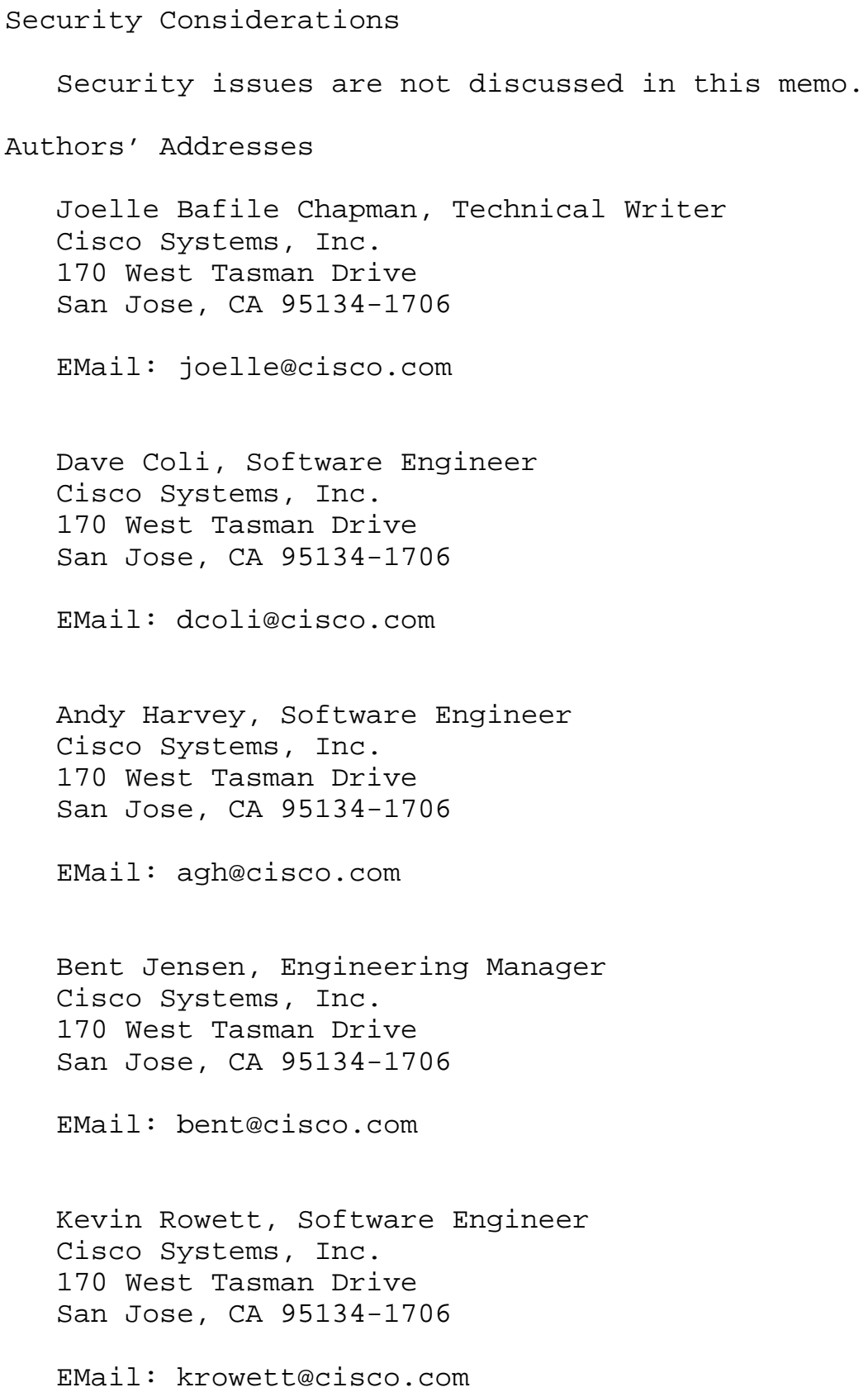

